Article

\title{
A Novel Real-Time Echo Separation Processing Architecture for Space-Time Waveform-Encoding SAR Based on Elevation Digital Beamforming
}

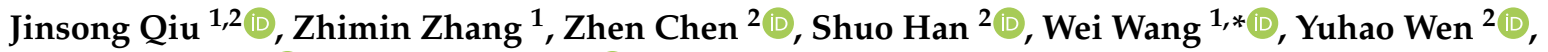 \\ Xiangrui Meng ${ }^{2} \mathbb{D}$ and Huaitao Fan ${ }^{1}$
}

Citation: Qiu, J.; Zhang, Z.; Chen, Z.; Han, S.; Wang, W.; Wen, Y.; Meng, X.; Fan, H. A Novel Real-Time Echo Separation Processing Architecture for Space-Time Waveform-Encoding SAR Based on Elevation Digital Beamforming. Remote Sens. 2022, 14, 213. https://doi.org/10.3390/ rs14010213

Academic Editors: Mi Wang, Hanwen Yu, Jianlai Chen and Ying Zhu

Received: 30 November 2021 Accepted: 31 December 2021 Published: 4 January 2022

Publisher's Note: MDPI stays neutral with regard to jurisdictional claims in published maps and institutional affiliations.

Copyright: (C) 2022 by the authors. Licensee MDPI, Basel, Switzerland. This article is an open access article distributed under the terms and conditions of the Creative Commons Attribution (CC BY) license (https:/ / creativecommons.org/licenses/by/ $4.0 /)$.
1 Department of Space Microwave Remote Sensing System, Aerospace Information Research Institute, Chinese Academy of Sciences, Beijing 100190, China; qiujingsong17@mails.ucas.edu.cn (J.Q.); zmzhang@mail.ie.ac.cn (Z.Z.); fanht@aircas.ac.cn (H.F.)

2 School of Electronic, Electrical and Communication Engineering, University of Chinese Academy of Sciences, Beijing 100190, China; chenzhen171@mails.ucas.ac.cn (Z.C.); hanshuo18@mails.ucas.ac.cn (S.H.); wenyuhao19@mails.ucas.ac.cn (Y.W.); mengxiangrui19@mails.ucas.ac.cn (X.M.)

* Correspondence: wwang@mail.ie.ac.cn

\begin{abstract}
Space-time waveform-encoding (STWE) SAR can receive echoes from multiple sub-swaths simultaneously with a single receive window. The echoes overlap each other in the time domain. To separate the echoes from different directions, traditional schemes adapt single-null steering techniques for digital receive beam patterns. However, the problems of spaceborne DBF-SAR, in practice, such as null extension loss, terrain undulation, elevation angle of arrival extension, and spaceborne antenna beam control, make the conventional scheme unable to effectively separate the echoes from different sub-swaths, which overlap each other in the time domain.A novel multi-null constrained echo separation scheme is proposed to overcome the shortcomings of the conventional scheme. The proposed algorithm can flexibly adjust the width of the notch to track the time-varying pulse extension angle with less resource consumption. Moreover, the hardware implementation details of the corresponding real-time processing architecture are discussed. The two-dimensional simulation results indicate that the proposed scheme can effectively improve the performance of echo separation. The effectiveness of the proposed method is verified by raw data processing instance of an X-band 16-channel DBF-SAR airborne system.
\end{abstract}

Keywords: synthetic aperture radar (SAR); digital beamforming (DBF); real-time processing; spacetime waveform-encoding (STWE)

\section{Introduction}

High-resolution wide swath (HRWS) imaging is a "development goal" for the next generation of spaceborne synthetic aperture radar (SAR) [1-3]. Owing to the inherent restrictions of the minimum SAR antenna area constraint, the traditional single-channel SAR cannot achieve a high-resolution image and wide swath simultaneously [4]. The mosaic mode [5] combines the spotlight mode with scan mode to counteract the degradation of azimuthal resolution in a traditional ScanSAR mode [6]. However, the mosaic mode does not fully meet the demand of next-generation spaceborne SAR for HRWS imaging. To overcome the minimum SAR antenna area constraint, the digital beamforming (DBF) technique is used to widen the imaging swath [7], or improve system performance, such as signal-to-noise ratio (SNR), noise equivalent sigma zero (NESZ), etc. The scan-on-receive (SCORE) mode based on DBF technology has been well studied [8] and experimented with [9]. On transmit, an equivalent wide (but low-gain) beam pattern is applied to cover the wide swath. Upon receive, an equivalent high gain and sharp digital receive beam pattern is formed along the varying direction of the received echo in real-time. 
The DBF imaging mode discussed in this paper is based on the space-time waveformencoding (STWE) mode [10,11], or intrapulse beam steering in elevation mode [12]. The STWESAR can realize waveform diversity in the space-time domain to meet the requirements of future HRWS missions. In the STWE architecture discussed in this paper, there is no restriction on the phase coding form and orthogonality of the signal being separated. Linear frequency modulation (LFM), nonlinear frequency modulation (NLFM), short-term shift-orthogonal (STSO), and other forms of signals can be separated using this scheme. Based on joint spacetime processing, the time-domain envelope and spatial information of the signal are of concern. To demonstrate the strength of the architecture proposed in this paper, a fully correlated LFM signal is used as a case study in the simulations and in the experiment sections. Since the signals have the same spectral characteristics, they can only be separated by subtle temporal and spatial differences. The principle of the discussed STWE-SAR is illustrated in Figure 1.

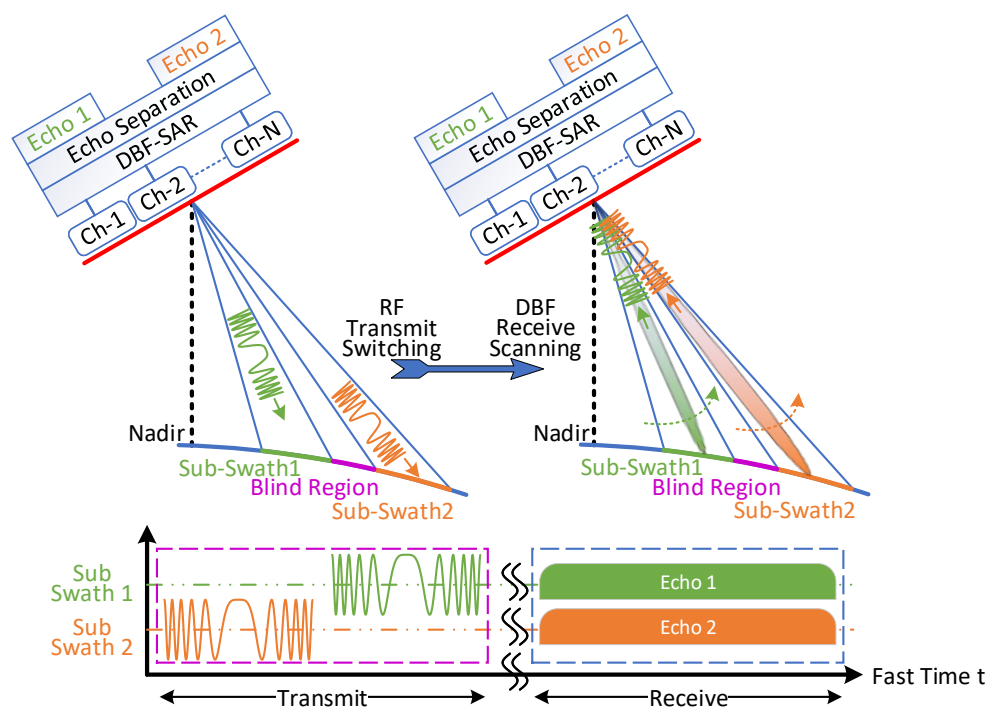

Figure 1. The principle diagram of a typical two-sub-swath STWE-SAR system.

Using two sub-swaths as an example, two wide imaging swaths are illuminated by two wide transmit beams, with a specific time sequence. The sub-swath in the far range is designed to be illuminated earlier than the sub-swath in the near range. The echo from the sub-swath in the far range always arrives at the antenna one pulse repetition time (PRT) later than the echo from the sub-swath in the near range. In this way, the echoes from different sub-swaths overlap each other in the time domain and, thus, the system can reduce the receiving window for echoes, which means breaking through the time constraints of traditional single channel SAR. Since each sub-swath is fully illuminated and received during the synthesis aperture imaging, a wider swath can be obtained without the decrease in azimuthal resolution. The Doppler history of each sub-swath is recorded in its entirety.

In a radar system, digital intermediate frequency (IF) receiver architecture is generally adopted [13]. On the receiver end, each channel amplifies, down converts (anti-aliasing filter follows), and digitizes the received signal by an analog-to-digital converter (ADC), obtaining the IF real signal of each channel, as illustrated in Figure 2. The data sample from each channel is weighted and summed in real-time by the field programmable gate array (FPGA) of a spaceborne DBF system. In the STWE-SAR hardware system, two parallel DBF modules are equipped to form two independent digital beam patterns in real-time. One DBF module steers the main beam to follow the echo from sub-swath 1 while suppressing the echo from sub-swath 2. Another DBF module receives echoes from sub-swath 2 and suppresses echoes from sub-swath 1 . 


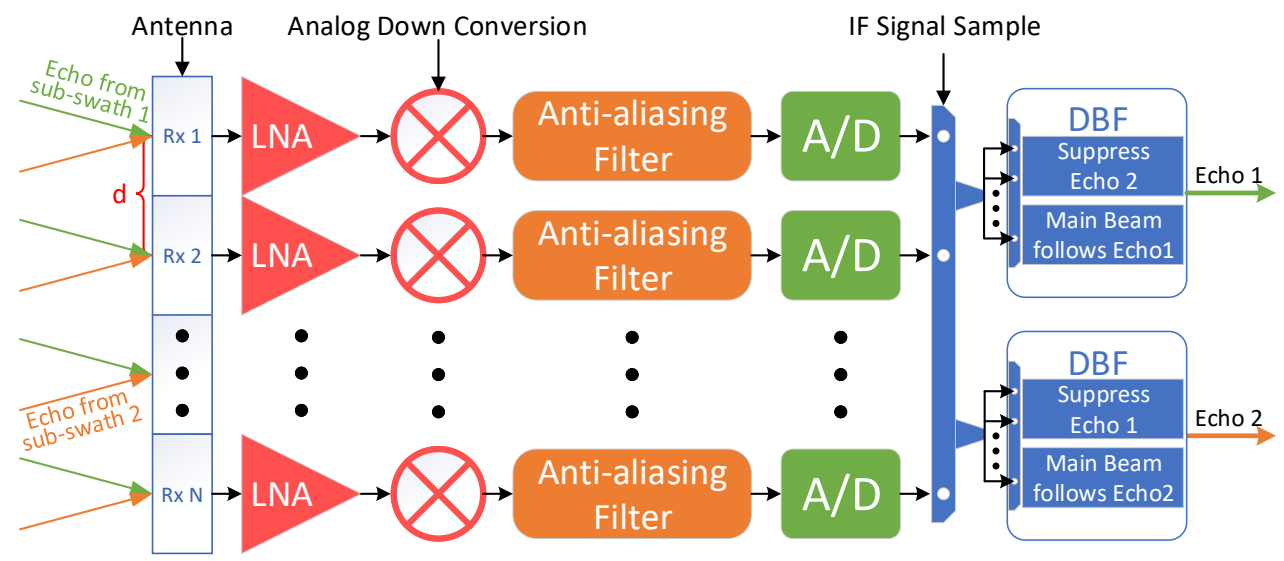

Figure 2. Block diagram of the top-level hardware structure of the STWE-SAR on receiver-end.

Since the DBF-SAR system is generally configured with multiple channels in elevation (e.g., TanDEM-L has 35 elements in elevation [1]), real-time DBF synthesis processing must be completed on-orbit and in real-time, otherwise the limited storage space onboard will limit the maximum coverage area of HRWS imaging. In order to realize real-time processing in spaceborne DBF-SAR, Feng et al. [14] provided a scheme configured with the finite impulse response (FIR) filter to suppress negative effects of the pulse extension loss (PEL) [15]. In order to reduce resource occupation, Wang et al. [16] proposed an improved processing architecture that requires only one quadrature demodulator in total. In order to guarantee the real-time performance of DBF-SAR, a weight generator for the SCORE mode at a low cost is proposed in [17], which utilizes an efficient approximate algorithm to calculate the weight. The real-time processing architecture proposed in this paper is based on previous work.

Since the space-time information of the so-called "interfering" signals is prior knowledge, the DBF spatial filter can be pre-designed and applied for real-time processing. The traditional STWE scheme steers one null of the pattern to follow and suppress the interference energy $[10,12]$. The one-dimensional (1D) simulation verifies the effectiveness of the conventional scheme $[10,11]$. However, some issues not observed in the 1D simulations will result in a large amount of interference energy that cannot be effectively suppressed. These issues are analyzed in Section 2.

A novel multi-null constrained scheme for STWE-SAR is proposed in this article. The main purpose is to overcome four significant problems of the conventional scheme. The proposed algorithm can flexibly adjust the width of the notch to track the time-varying pulse extension angle with less resource consumption. A digital beam pattern with a wider low-level gain notch is formed to suppress the interference echo from one specific direction for a long time while the beam pattern keeps steering. Therefore, the effective suppression of interference has been greatly enhanced. The derivation of the algorithm is given and details of the real-time processing implementation are discussed. The proposed weight generator utilizes an efficient approximate algorithm to calculate the weight in real-time. Furthermore, the digital array steering and scan loss compensation are discussed.

\section{Existing Problems in the Traditional Scheme}

A DBF-SAR system with $N$ channels in elevation can form a sharp and high-gain receiving beam along the varying direction of the received echo in real-time, which scans from the near-end to far-end of the swath, as illustrated in Figure 3. 


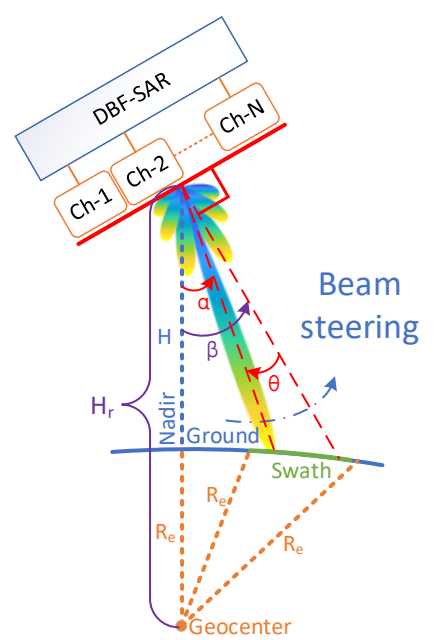

Figure 3. Imaging geometry of a DBF-SAR system.

In this article, the default reference channel is channel 1 , which is at the far right end of the array. Term $H$ represents the height of the orbit. Term $\theta$ and $\beta$ denote the broadside angle and the antenna's normal direction, respectively. The time-varying look angle $\alpha(t)$ can be derived from the geometry of Figure 3 as follows:

$$
\alpha(t)=\operatorname{acos}\left(\frac{H_{r}^{2}+r(t)^{2}-R_{e}^{2}}{2 \cdot H_{r} \cdot r(t)}\right)
$$

where $H_{r}$ and $R_{e}$ represent the distance from a satellite to the center of the Earth and the radius of the Earth, respectively. Term $r(t)$ denotes the slant range and can be written as

$$
r(t)=c \frac{\left(t+T_{0}\right)}{2}, t \in\left[-\frac{P R T}{2}, \frac{P R T}{2}\right]
$$

where $T_{0}$ and $c$ denote the round trip delay corresponding to the center of the scene and the speed of light, respectively. Term $t$ denote the fast time. In the traditional STWE scheme, time-varying weights control one null of the pattern to follow and suppress the interference energy. However, four significant problems in the traditional singlenull scheme can substantially weaken the suppression of interfering energy in the actual working environment of a spaceborne SAR.

The first and most important issue is related to pulse extension loss (PEL). A narrow beam steering toward a pulse echo will cause PEL [15]. Since the width of the null is much shorter than the main lobe, a null steering toward a pulse will certainly cause an effect, such as the PEL, which is named Null Extension Loss (NEL) in this paper. The principle and impact of the NEL and the PEL are shown in Figure 4.

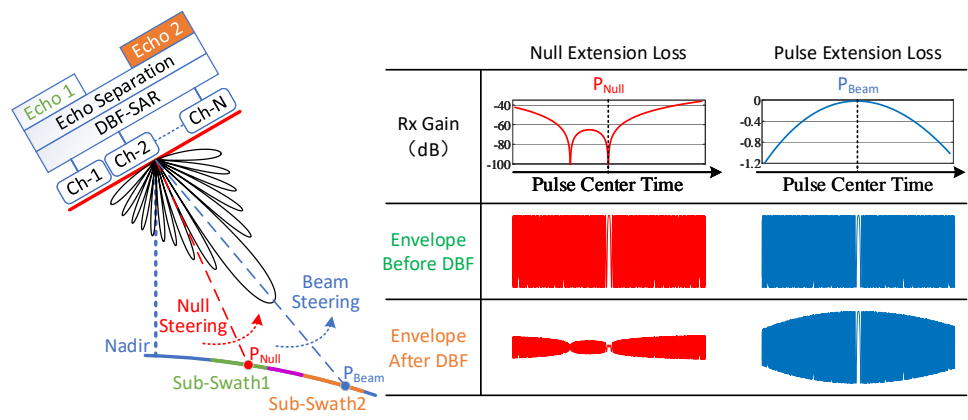

Figure 4. Diagram of the PEL and the NEL effects. 
According to [18], the time-varying angular pulse extent gives the analytic expression of $\chi_{\alpha}(t)$, which is written as

$$
\chi_{\alpha}(t)=\frac{T_{r} c}{2 H_{r}\left(\frac{H_{r} \cdot \cos (\alpha(t))}{\sqrt{R_{e}^{2}-\left(H_{r} \cdot \sin (\alpha(t))\right)^{2}}}-1\right) \sin (\alpha(t))}
$$

where $T_{r}$ denotes the pulse width. Referring to the definition of PEL in [15], the following definition of the NEL is formulated:

$$
N E L(\alpha)=-\frac{1}{\chi_{\alpha}} \int_{\alpha_{0}-\chi_{\alpha} / 2}^{\alpha_{0}+\chi_{\alpha} / 2}\left|C_{R x}\left(\alpha, \alpha_{0}\right)\right|^{2} d \alpha
$$

where $C_{R x}$ represents the receiving antenna pattern formed by the STWE-SAR system.

The STWE-SAR system steers the main lobe and the null of the digital received beam pattern together. The main lobe and the null follow the pulse center of echo signals to receive energy in the direction of interest and suppress interference energy simultaneously. As can be seen in Figure 4, the echoes will be modulated by the envelope of the digital beam. The energy at the center of the pulse echo from $P_{\text {Null }}$ is completely suppressed, while the energy at the center of the pulse echo from $P_{\text {Beam }}$ is retained well.

The second issue is related to the effect of undulating terrain on the DBF receive performance. As shown in Figure 5, in HRWS imaging, the undulating terrain causes the arrival direction of the echoes to deviate from the angle calculated in advance based on the geometry model. The width of the main lobe enables the pattern to tolerate slight topographic relief at the cost of slight gain loss. However, the width of the null is so narrow that even slight topographic relief will lead to a significant reduction of interference suppression ability. Therefore, terrain undulation is trouble for the single-null interference suppression scheme in the conventional STWE scheme.
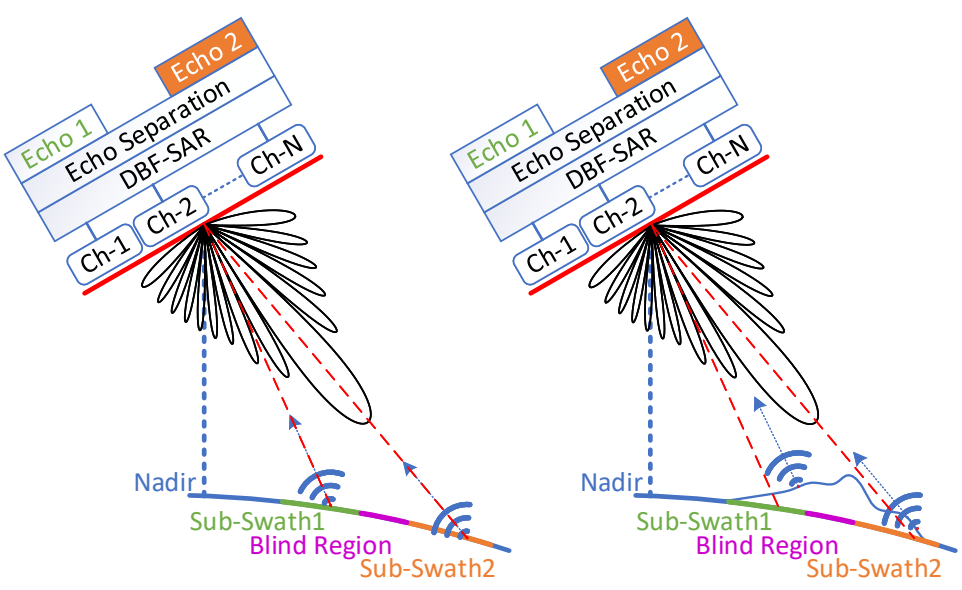

Figure 5. Diagram of the the effect of undulating terrain on the DBF reception performance.

The third issue is related to the spatial distribution of echo. In the 1D simulations of $[10,11]$, the simulation results of the conventional STWE scheme are satisfactory. However, a phenomenon not observed in the 1D simulations will result in a large amount of interference energy that cannot be effectively suppressed. In this paper, this phenomenon is named an elevation angle of arrival (AoA) extension. The geometry of the elevation AoA extension phenomenon is shown in Figure 6. 


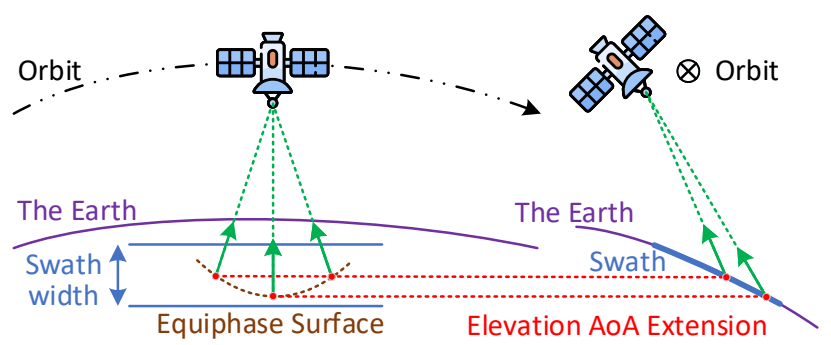

Figure 6. The geometry diagram of the elevation AoA extension phenomenon.

The spherical wave emitted by the radar antenna propagates in the space toward the ground, and the boundary where the wave front intersects the ground, is known as the equiphase surface. All targets on the same equiphase surface are at different look angles. In other words, a set of signals from different elevation angles will arrive at the receiving antenna at the same time. It is impossible to suppress all signals in the extent shown in Figure 6, with a narrow null. The elevation AoA extension effect will cause most of the interfering signal to deviate from the null direction. Only the interfering energy from the light of sight (LOS) direction will be suppressed by the null at an ultra-low level, while most of the interfering energy will be received by the pattern's side lobe.

The last issue is related to array steering. For spaceborne SAR, increasing the area of the antenna can effectively improve the performance of the antenna. The DBF-SAR system relies on the scanning capability of the multi-channel array to enhance the SNR for wide swath imaging. Without increasing the number of channels, a larger total antenna area will result in a larger spacing between channels. However, the grating lobes of the beam pattern may limit the scanning capability of the array [19]. In general, we require

$$
\frac{d}{\lambda} \leq \frac{1}{1+\left|\sin \bar{\theta}_{\max }\right|}
$$

where $\bar{\theta}_{\text {max }}$ denotes the maximum angle to which the array will be required to steer. Term $\lambda$ and $d$ represent the wavelength that corresponds to the carrier frequency and the element spacing of the array. Fortunately, by adjusting the latency of the programmable delay and amplification components in the TR module (TRM) [20], equivalent large-angle scanning can be achieved. As shown in Figure 7, the equivalent antenna boresight is adjusted, a large angle $\varphi$ with the help of TRM. Based on above, the DBF module only needs to steer the beam in a small angle region to avoid severe grating lobes in the beam pattern.

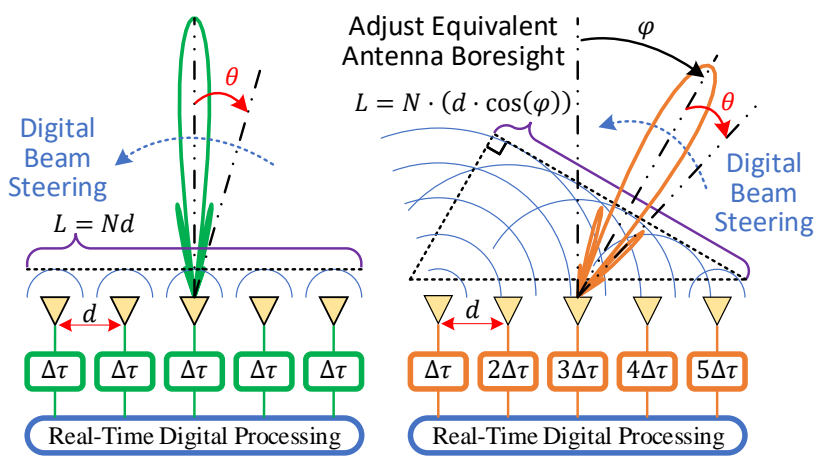

Figure 7. Diagram of the receiving beam control of the antenna.

At any time, the TRM can only drive the main response axis (MRA) to align with one specified beam position. However, in STWE imaging mode, the system must control a set of digital beams to scan two or more different sub-swaths simultaneously. In order to achieve real-time processing - how to replace the original analog delay elements with parallel digital delay elements will be discussed in Section 3. 


\section{Proposed Algorithm and Real-Time Processing Architecture}

The beam pattern formed by the conventional STWE imaging mode is a single-null constraint pattern as shown in Figure 8a. As previously discussed in Section 2, the single null constraint scheme has unsatisfactory performance in suppressing interference because of the inherent characteristics and spatial distribution of the echo. In order to overcome the shortage of interference energy suppression ability of the traditional scheme, the proposed algorithm and processing architecture are applied to generate a multi-null constraint beam pattern as illustrated in Figure $8 \mathrm{~b}$ in real-time. When the number of null constraints is greater than one, a digital beam pattern with a wider low-level gain interference suppression notch can be formed.

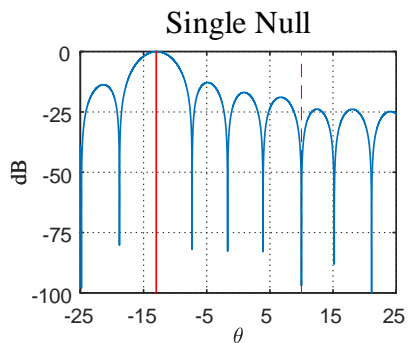

(a)

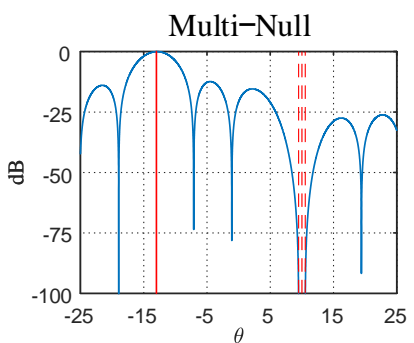

(b)

Figure 8. (a) A single null constrained beam pattern. (b) A multi-null constrained beam pattern.

\subsection{Principle and Algorithm Derivation}

A beam pattern with a wide notch can suppress the interference echo from one specific direction for a long time while the beam pattern keeps steering. As shown in Figure 9, the echo from point $P_{N u l l}$ is always inside the notch during the time when the pulse is received. The notch extent that can completely cover the echo from a fixed look angle is called the pulse extension angle.

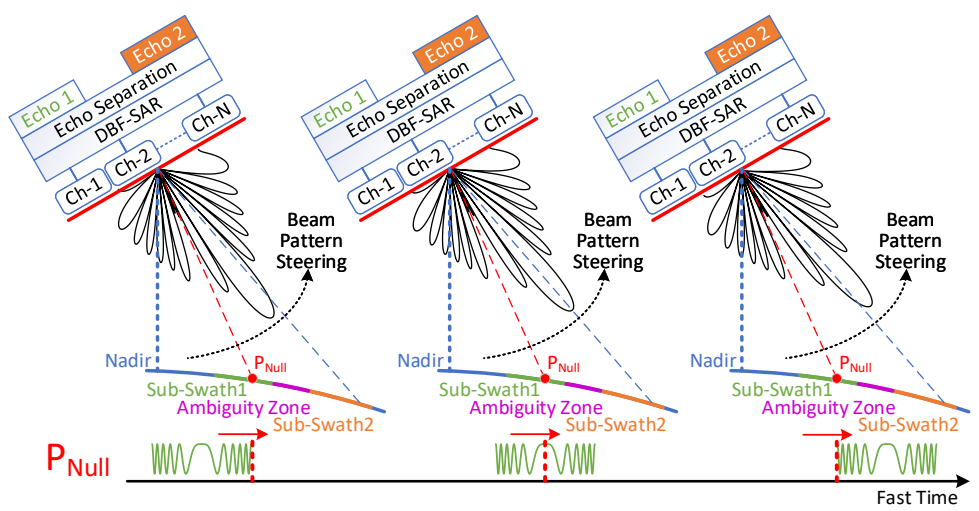

Figure 9. The principle diagram of the proposed method.

Since the Earth's surface has a curvature, the pulse extension angle corresponding to the echoes from different look angles is not fixed. As shown in Figure 10, the pulse extension angle at the far end is smaller than the pulse extension angle at the near end. The border of the pulse extension angle also shrinks inward as the slant range increases. Therefore, a beam pattern with a fixed-width notch is not fully satisfactory for the STWE imaging mode. The algorithm proposed in this section can adaptively adjust the width of the notch with less resource consumption to minimize the impact caused by the NEL and other factors discussed in Section 2. 


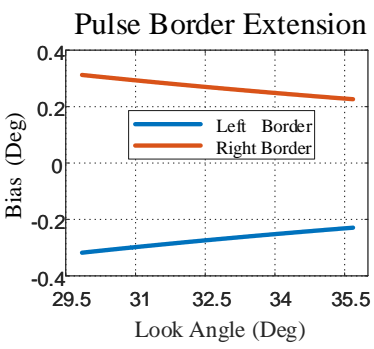

(a)

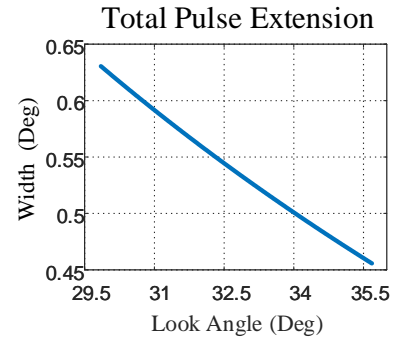

(b)

Figure 10. (a) The border of the pulse extension angle. (b) The pulse extension angle.

Considering a narrowband $\left(B_{r} / f_{0} \ll 1\right)$ DBF-SAR system with $N$ equidistant channels in elevation, the DBF weights are time-varying in fast time. For a single snapshot, the DBF processing can be modeled as

$$
\left\{\begin{array}{l}
Y=w^{H} \boldsymbol{X} \\
\boldsymbol{X}=S_{d} \boldsymbol{V}+S_{\boldsymbol{n}}
\end{array}\right.
$$

where $S_{d}$ and $Y$ represent the directed signal before DBF synthesis and the signal after DBF synthesis, respectively; and $X$ and $S_{n}$ represent the vector of observed multichannel received signal and the vector of thermal noise, respectively. $X \in \mathbb{C}^{N \times 1}$, and $S_{n} \in \mathbb{C}^{N \times 1}$. Term $\boldsymbol{w}^{H}$ denotes the vector of DBF weights generated in real-time. $\boldsymbol{w}^{H} \in \mathbb{C}^{1 \times N}$.

Term $V$ refers to the array manifold vector. $V \in \mathbb{C}^{N \times 1}$.

$$
\boldsymbol{V}(\theta)=\left[\begin{array}{c}
\exp \left(-j 2 \pi\left(\frac{N-1}{2}\right) \frac{d}{\lambda} \sin (\theta)\right) \\
\exp \left(-j 2 \pi\left(\frac{N-3}{2}\right) \frac{d}{\lambda} \sin (\theta)\right) \\
\vdots \\
\exp \left(j 2 \pi\left(\frac{N-3}{2}\right) \frac{d}{\lambda} \sin (\theta)\right) \\
\exp \left(j 2 \pi\left(\frac{N-1}{2}\right) \frac{d}{\lambda} \sin (\theta)\right)
\end{array}\right]
$$

where $j$ and $\theta$ represent the imaginary unit and the broadside angle, respectively. The beam pattern is calculated by

$$
B(\theta)=\boldsymbol{w}^{H} \boldsymbol{V}(\theta) \quad, \quad \theta \in\left[-\frac{\pi}{2}, \frac{\pi}{2}\right]
$$

The well-known minimum variance distortionless response (MVDR) beamforming method requires solving the correlation matrix of the signal sample, which is difficult to be implemented in real-time processing. In order to facilitate multi-null constraint and realtime computation, it is recommend to use linear constrained minimum variance (LCMV) as the optimization algorithm in the real-time processing architecture. The multi-null optimization model for STWE-SAR system can hence be established by

$$
\left\{\begin{array}{cl}
\min _{w} & \boldsymbol{w}^{H} \boldsymbol{R}_{\boldsymbol{n}} \boldsymbol{w} \\
\text { s.t. } & \boldsymbol{w}^{H} \boldsymbol{C}=\boldsymbol{e}^{H}
\end{array}\right.
$$

where $\boldsymbol{R}_{\boldsymbol{n}}$ represents spectral matrix of the thermal receiver noise [19]. Assuming that the noise is white with the power $\sigma_{n}^{2}$ [10], $\boldsymbol{R}_{n}$ can be expressed as

$$
\boldsymbol{R}_{n}=\sigma_{n}^{2} \cdot \mathbf{I}
$$

where I represent the identity matrix, $\mathbf{I} \in \mathbb{C}^{N \times N}$. 
Term $C$ represents the constraint matrix containing 1-order distortionless constraint and a $Q$-order null constraint, $C \in \mathbb{C}^{N \times(Q+1)}$.

$$
C=\left[V_{\text {Beam }}, V_{\text {Null }}\right]
$$

and $\boldsymbol{e}^{H}$ denotes the constraint vector, $\boldsymbol{e}^{H} \in \mathbb{R}^{1 \times(Q+1)}$.

$$
\boldsymbol{e}^{H}=[1,0, \ldots, 0]
$$

Term $V_{\text {Beam }}$ represents the array manifold vector in the direction of the distortionless, $V_{\text {Beam }} \in \mathbb{C}^{1 \times M}$. The main lobe is guided to $\theta_{b}$.

$$
\boldsymbol{V}_{\text {Beam }}=\left[\begin{array}{c}
\exp \left(-j 2 \pi\left(\frac{N-1}{2}\right) \frac{d}{\lambda} \sin \left(\theta_{b}\right)\right) \\
\exp \left(-j 2 \pi\left(\frac{N-3}{2}\right) \frac{d}{\lambda} \sin \left(\theta_{b}\right)\right) \\
\vdots \\
\exp \left(j 2 \pi\left(\frac{N-3}{2}\right) \frac{d}{\lambda} \sin \left(\theta_{b}\right)\right) \\
\exp \left(j 2 \pi\left(\frac{N-1}{2}\right) \frac{d}{\lambda} \sin \left(\theta_{b}\right)\right)
\end{array}\right]
$$

Term $V_{\text {Null }}$ represents the array manifold matrix of the $Q$-order null constraint, $V_{\text {Null }} \in$ $\mathbb{C}^{N \times Q}$. The pattern has $Q$ zeros in the direction of $\left[\theta_{0_{1}}, \theta_{0_{2}}, \ldots, \theta_{0_{\mathrm{Q}}}\right]$.

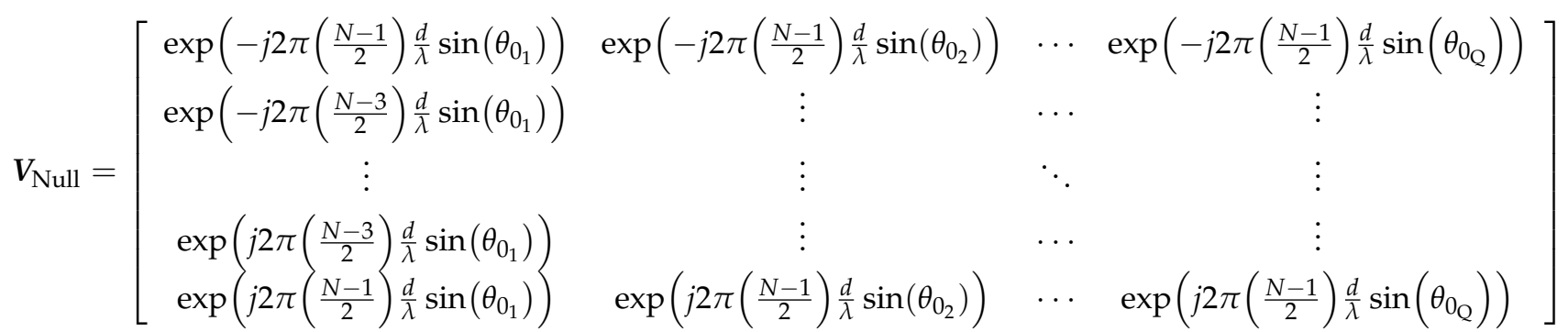

Using the LCMV beamforming method [19], one can derive the $\boldsymbol{W}_{\mathrm{lcmv}}^{\mathrm{H}}$ as

$$
\boldsymbol{W}_{\mathrm{lcmv}}^{\mathrm{H}}=\boldsymbol{e}^{\mathrm{H}}\left[\boldsymbol{C}^{\mathrm{H}} \boldsymbol{R}_{n}^{-1} \boldsymbol{C}\right]^{-1} \boldsymbol{C}^{\mathrm{H}} \boldsymbol{R}_{n}^{-1}
$$

Substituting (10) into this expression, (15) can be simplified as:

$$
W_{\mathrm{lcmv}}^{\mathrm{H}}=e^{\mathrm{H}}\left[C^{\mathrm{H}} C\right]^{-1} C^{\mathrm{H}}
$$

It is worth noting that the first column of the weights $W_{\mathrm{lcmv}}^{\mathrm{H}}$ corresponds to the leftmost element of the array. For the array sequence in Figure 3 (channel 1 near the ground), the first column of $\boldsymbol{W}_{\mathrm{lcmv}}^{\mathrm{H}}$ corresponds to the weights of channel $N$ while the last column corresponds to the weights of channel 1.

The time-varying weights $\boldsymbol{W}_{\mathrm{lcmv}}^{\mathrm{H}}$ are obtained by calculating the constraint matrix $C$ in real-time onboard. The approximate algorithm proposed in [17] can be applied to calculate time-varying phase terms of the constraint matrix $C$. The time-varying phase can be expressed as

$$
\varphi_{n}(t)=-A F_{n} \cdot f(t)
$$


The negative sign introduced here is due to the fact that the channel 1 is at the right end of the array in this paper. Term $A F_{n}$ represents the array factor, which is expressed as

$$
A F_{n}=2 \pi\left(n-\frac{N+1}{2}\right) \frac{d}{\lambda}
$$

According to [17], although the expression of $f(t)$ is complicated, the function image of $f(t)$ is a simple and monotonically increasing arc. Therefore, function $f(t)$ can be well-approximated by using only a few terms of its Taylor series.

The Taylor series of function $f(t)$ at $t=0$ is expressed as

$$
f(t)=\sum_{k=0}^{k=M} \frac{f^{k^{\prime}}(0)}{k !}\left(t^{k}\right)+o\left(t^{M}\right)
$$

where $k$ ! denotes the factorial of $k$. Term $o\left(t^{M}\right)$ is called the Peano form of the remainder, which represents an approximation error when $f(t)$ is approximated with its Taylor polynomial. The Taylor series of $f(t)$ helps to find the closed-form expression of parameters $\left\{A_{0}, A_{1}, \ldots, A_{M}\right\}$. Then, (19) can be rewritten as

$$
f(t)=\sum_{k=0}^{k=M} A_{k}(t)^{k}+o\left(t^{M}\right)
$$

Now, an M-order linear polynomial $g(t)$ based on parameters $\left\{A_{0}, A_{1}, \ldots, A_{M}\right\}$ can be used to approximate the original function $f(t)$ as follows

$$
g(t)=A_{0}+A_{1} \cdot t+A_{2} \cdot t^{2}+\ldots+A_{M} \cdot t^{M} \approx f(t)
$$

Although the expressions of parameters $\left\{A_{0}, A_{1}, A_{2}, \ldots\right\}$ are somewhat sophisticated, they do not need to be calculated in real-time [17]. These parameters can be calculated in advance by an auxiliary computer.

Since the closed-form expression of the submatrix $V_{\text {Beam }}$ and $V_{\text {Null }}$ of matrix $C$ is similar. The polynomial approximation method can be used to calculate $V_{\text {Beam }}$ in real-time. We have

$$
V_{\text {beam }}(t) \approx \exp \left(-j\left[\begin{array}{c}
A F_{1} \cdot g_{b}(t) \\
\vdots \\
A F_{n} \cdot g_{b}(t) \\
\vdots \\
A F_{\mathrm{N}} \cdot g_{b}(t)
\end{array}\right]\right)
$$

where $g_{b}(t)$ denotes the linear polymeric approximation of the time-varying function of the main lobe beam constraint.

$$
g_{b}(t) \approx f_{b}(t)=\sin \left(\alpha_{\text {beam }}(t)-\beta\right)
$$

where $\alpha_{\text {beam }}(t)$ represents the direction of arrival of the echo signal pulse center at time $t$. $V_{\text {Beam }}$ leads the main lobe to follow the time-varying direction to obtain high gain receiving of the echo. With the linear polynomial approximation shown in Equation (21), the complexity of the onboard implementation is greatly simplified.

To minimize the impact caused by the NEL and other factors, the proposed algorithm arranges multiple constrained nulls to form a digital beam pattern with a wide notch, to suppress the extension energy of the pulse in the spatial domain. However, as shown in Figure 10, the width of the pulse spread varies with the look angle. Calculating the 
boundary of the notch $\theta_{\text {pulse }}$ based on the spatial relationship [18] between the pointing angles of the null points will increase the complexity of the system.

$$
\theta_{\text {pulse }}=\frac{T_{r} c}{2 H_{r}\left(\frac{H_{r} \cdot \cos (\alpha(t))}{\sqrt{R_{e}^{2}-\left(H_{r} \cdot \sin (\alpha(t))\right)^{2}}}-1\right) \sin (\alpha(t))}
$$

The proposed method exploits the relationship between the space-time of the echo to simplify the calculation complexity of the constraints. The notch of the pattern is formed by a multi-null constraint, where the two outermost points determine the width of the notch. Since the interference echo also follows the geometric relationship given in Equation (1), the arrival direction $\alpha_{\mathrm{INF}}(t)$ of the pulse center of the interference echo can be calculated and then used to generate the multi-null constraint. In the proposed method, the timing constraints between the $Q$ nulls satisfy the following conditions.

$$
\left[\begin{array}{c}
\theta_{0_{1}}(t) \\
\vdots \\
\theta_{0_{\mathrm{q}}}(t) \\
\vdots \\
\theta_{0_{\mathrm{Q}}}(t)
\end{array}\right]=-\left[\begin{array}{c}
\alpha_{\mathrm{INF}}\left(t-\frac{T_{r}}{2}\right)-\beta \\
\vdots \\
\alpha_{\mathrm{INF}}\left(t-\left(q-\frac{Q+1}{2}\right) T_{r}\right)-\beta \\
\vdots \\
\alpha_{\mathrm{INF}}\left(t+\frac{T_{r}}{2}\right)-\beta
\end{array}\right]
$$

Null points on the beam pattern in the $\theta_{0_{1}}$ and $\theta_{0_{Q}}$ directions are aimed at the arrival direction at the start and end time of the pulse echo, respectively. The multi-null constrained beam pattern allows the echo pulse to be received in the coverage of the notch during the pulse time. The multi-null constrained notch scheme not only reduces the impact of NEL on the system, but also suppresses interference over a wide range, much better than the traditional single-null scheme.

$V_{\text {Null }}$ can be expressed as a combination of array factors and time-varying functions. The time-varying function of $\boldsymbol{V}_{\text {Null }}$ can be written as

$$
\left\{\begin{array}{c}
f_{0_{1}}(t)=\sin \left(\alpha_{\mathrm{INF}}\left(t-\frac{T_{r}}{2}\right)-\beta\right) \\
\vdots \\
f_{0_{\mathrm{Q}}}(t)=\sin \left(\alpha_{\mathrm{INF}}\left(t+\frac{T_{r}}{2}\right)-\beta\right)
\end{array}\right.
$$

To simplify the expression of the $C$ matrix, let

$$
\left\{\begin{array}{c}
g_{0_{1}}(t)=g_{0}\left(t-\frac{T_{r}}{2}\right) \\
\vdots \\
g_{0_{Q}}(t)=g_{0}\left(t+\frac{T_{r}}{2}\right)
\end{array}\right.
$$

where

$$
g_{0}(t) \approx \sin \left(\alpha_{\mathrm{INF}}(t)-\beta\right)
$$

For a single snapshot, the expression for the $C$ matrix can be written as

$$
\boldsymbol{C}=\exp \left(-j\left[\begin{array}{cccc}
A F_{1} g_{b} & A F_{1} g_{0_{1}} & \cdots & A F_{1} g_{0_{\mathrm{Q}}} \\
A F_{2} g_{b} & A F_{2} g_{0_{1}} & \cdots & A F_{2} g_{0_{\mathrm{Q}}} \\
\vdots & \vdots & \ddots & \vdots \\
A F_{\mathrm{N}} g_{b} & A F_{\mathrm{N}} g_{0_{1}} & \cdots & A F_{\mathrm{N}} g_{0_{\mathrm{Q}}}
\end{array}\right]\right)
$$




\subsection{Digital Array Steering}

In the conventional scheme, TRMs adjust the equivalent antenna boresight by configuring the latency between different channels. However, in STWE mode, the simultaneous receiving of multiple sub-swath echoes means that several different equivalent antenna boresight are needed. In the proposed DBF real-time processing architecture, a set of parallel digital delay components replaces the conventional analog devices in TRMs to adjust MRA.

The steering angle can be expressed as

$$
\phi=\alpha_{c}-\beta
$$

where $\alpha_{c}$ and $\beta$ denote look angle center of the interested sub-swath and the antenna's normal direction, respectively. In 1D array case, the initial coordinate vector of the $n$th array element can be represented as

$$
\boldsymbol{P}_{n_{\text {Init }}}=\left[\begin{array}{c}
\left(n-\frac{N+1}{2}\right) d \\
0
\end{array}\right]
$$

The coordinate matrix of the $n$th array element after rotation can be represented as

$$
\boldsymbol{P}_{n_{\text {Rot }}}=\mathcal{R}(\phi) \boldsymbol{P}_{n_{\text {Init }}}
$$

where $\mathcal{R}(\phi)$ denotes the rotation matrix.

$$
\mathcal{R}(\phi)=\left[\begin{array}{cc}
\cos (\phi) & -\sin (\phi) \\
\sin (\phi) & \cos (\phi)
\end{array}\right]
$$

The latency of the $n$th channel can be represented as

$$
\mathcal{D}_{n}=-\operatorname{sgn}\left(A F_{n}\right) \cdot \frac{\left\|\boldsymbol{P}_{n_{\mathrm{Rot}}}-\boldsymbol{P}_{n_{\text {Init }}}\right\|_{2}}{c}
$$

where $A F_{n}$ is the array factor in (18). Term $\operatorname{sgn}(\bullet)$ represents the signum function. The negative sign introduced here is due to the fact that the channel 1 is at the right end of the array in this paper. Term $\|\bullet\|_{2}$ represents the two-norm of a vector that is used to calculate the virtual displacement, due to the rotation of the array element.

An IF-DBF real-time processing architecture was proposed in [16] and improved by combining the real-time weight generator in [17]. As illustrated in Figure 11, signals are delayed for $\Delta t_{n}$ by the FIR filter to compensate PEL. This FIR filter performs a dispersive. It can be regarded as an interpolator to obtain the required $\Delta t_{n}$.

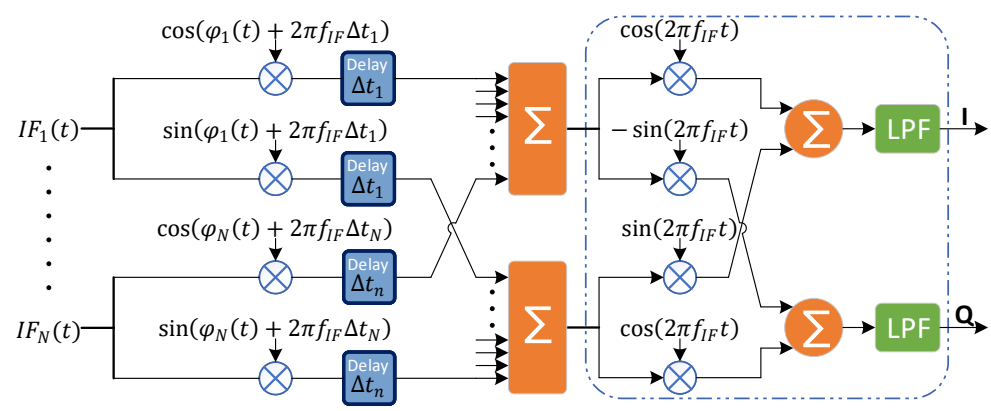

Figure 11. Block diagram of the IF-DBF architecture. 
All the latencies can be implemented together in the real-time processing framework shown in Figure 11. The new delay value $\Delta t_{n}{ }^{\prime}$ of the $n$th channel can be rewritten as

$$
\Delta t_{n}{ }^{\prime}=\mathcal{D}_{n}-\Delta t_{n}
$$

Since the digital delay component in Figure 11 is placed after the weighting component, the digital beam pattern formed by the algorithm mentioned in the previous section is based on the original antenna's normal direction. The delayed signals and digital pattern are adjusted by the center of the sub-swath as a reference. The maximum scan angle of each digital beam is reduced to half of the sub-swath's look angle extent. The adjustment will partially compensate for the scan loss due to the large scan angle.

\subsection{Real-Time Implementation}

Reducing the complexity and resource consumption of onboard processing implementations is the subject of the following discussion. Since the array factor given by (18) has symmetry, the consumption of real-time calculation resources for the $C$ matrix can be reduced by half, based on row symmetry. When the number of channels $N$ is even, the $C$ matrix can be split into two symmetric submatrices. When the number of channels $N$ is odd, the middle of the $C$ matrix is a row array of all ones.

$$
C=\left\{\begin{array}{l}
{\left[\begin{array}{c}
C_{S} \\
\cdots \\
\cdots \\
\mathrm{J} C_{S}^{*}
\end{array}\right], \quad N \text { is odd }} \\
{\left[\begin{array}{c}
C_{S} \\
\hdashline C_{S}^{*}
\end{array}\right], \quad N \text { is even }}
\end{array}\right.
$$

where

$$
C_{S}=\exp \left(-j\left[\begin{array}{cccc}
A F_{1} g_{\mathrm{b}} & A F_{1} g_{1} & \cdots & A F_{1} g_{\mathrm{Q}} \\
A F_{2} g_{\mathrm{b}} & A F_{2} g_{1} & \cdots & A F_{2} g_{\mathrm{Q}} \\
\vdots & \vdots & \ddots & \vdots \\
A F_{k} g_{\mathrm{b}} & A F_{k} g_{1} & \cdots & A F_{k} g_{\mathrm{Q}}
\end{array}\right]\right)
$$

where

$$
\begin{cases}k=\frac{N-1}{2}, & N \text { is odd } \\ k=\frac{N}{2}, & N \text { is even }\end{cases}
$$

Term $\mathrm{JC}_{S}^{*}$ represents the conjugate matrix after the row symmetric transformation of $C_{S}$.

$$
\mathrm{J} C_{S}^{*}=\operatorname{conj}\left(\mathbf{J} \cdot C_{S}\right)
$$

where $\mathbf{J}$ represents the exchange matrix. The definition of $\mathbf{J}$ is given in Appendix A. The row transformation can be implemented by rearranging the internal wires of the FPGA without any additional calculations.

The resource consumption of implementing the $C^{H}$ matrix can also be reduced by symmetry.

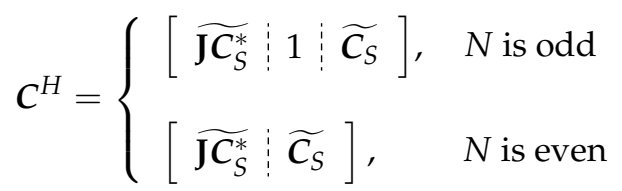


where $\widetilde{(\bullet)}$ denotes the rotation of the matrix clockwise by 90 degrees. The derivation process is given in Appendix B. Replacing part of the calculation by rearranging the internal wires of the FPGA is the key to reduce the calculation resources in the proposed method. In order to calculate the trigonometric function by phase $\varphi_{n}(t)$ in real-time, the COordinate Rotation DIgital Computer (CORDIC) algorithm, proposed by J.E. Volder in 1959 [21] should be considered. Approximate values of $\cos \left(\varphi_{n}(t)\right)$ and $\sin \left(\varphi_{n}(t)\right)$ can be calculated at the same time after a finite number of iterations.

The block diagram of the proposed constraint matrix generator is as presented in Figure 12. By multiplying the array factor $A F_{n}$ and the approximate linear polynomial $g$ of the constraint functions of the main lobe and multi-null, the time-varying phase of the constraint matrix $C_{S}$ can be obtained. Then, the complex value of the matrix $C_{S}$ is calculate by the CORDIC IP core, and the matrix $\mathrm{JC}_{S}^{*}$ is obtained by row transformation and conjugation. Finally, the $C$ and $C^{H}$ are obtained by splicing the outputs through FPGA wiring.

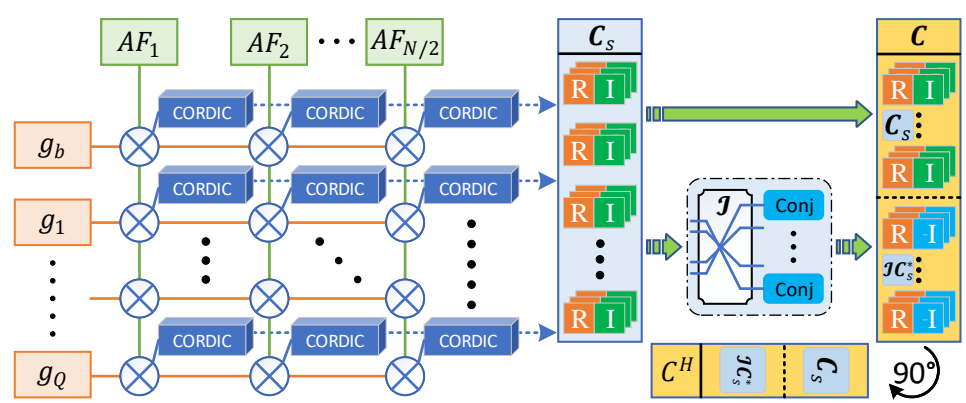

Figure 12. The block diagram of the constraint matrix generator.

The total number of real number multipliers consumed by matrices $C$ and $C^{H}$ is given by

$$
\mathrm{NUM}_{\mathrm{R}_{\mathrm{MUL}}}=\frac{N}{2} \times(1+Q)
$$

The LCMV beamformer needs to calculate the inverse matrix in real-time onboard. The size of the inverse matrix is only related to the number of null constraints, without regard to the number of channels. The beam pattern with more null constraints has better suppression performance against interference and require more resources to implement. The effect of a different number of null constraints on the directional map will be analyzed in Section 4. A minimum of no less than three null constraints are recommended to suppress the beginning, middle, and end of the interference pulse, respectively. Therefore, the minimum size of the inverse matrix required in the two-sub-swath STWE-SAR system is $4 \times 4$, which can be implemented on a single Xilinx Virtex-4 FPGA. It is based on XtremeDSP block, block RAM, and CORDIC core, all provided by the Xilinx CORE Generator toolset [22].

As illustrated in Figure 13, the architecture of the proposed weight generator is used to calculate (16) in real-time. At the end of the module, a set of complex multipliers introduce a constant modulation $\exp (j \psi)$ to the weights. In [16], the weights are appended with factors used to compensate the PEL in IF-DBF architecture. In addition, the compensation factors for the amplitude and phase errors can also be corrected by the same set of complex multipliers. 


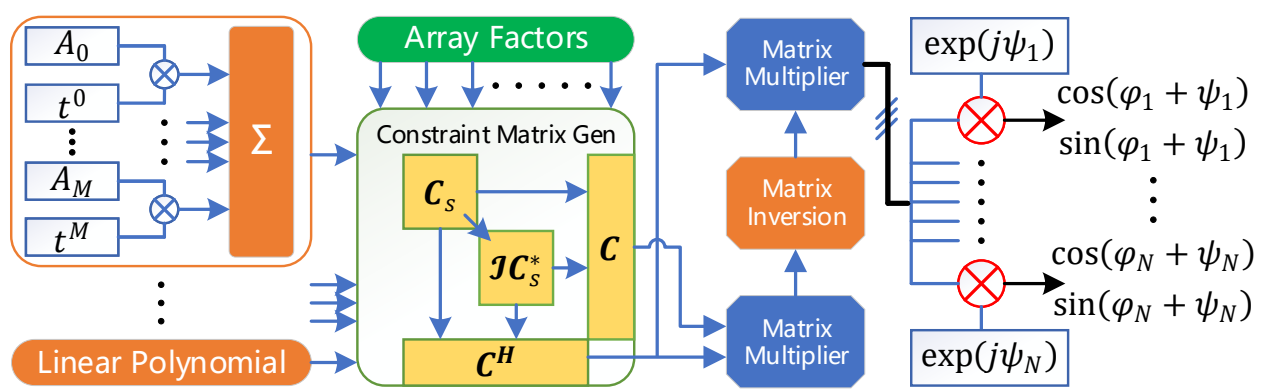

Figure 13. The block diagram of the proposed real-time weight generator for STWE-SAR system.

Assume that the matrix multiplier in Figure 13 is a timing logic circuit, consisting of a row of multipliers of length $N$ and some accumulators. This type of matrix multiplier can only get the result of multiplying two matrices when all rows of the matrix have been computed. The computational latency reduces the update rate of the weights. Discussions on weight update rates are given in $[17,23]$. The total number of complex number multipliers consumed by the proposed real-time weight generator illuminated in Figure 13 is given by

$$
\mathrm{NUM}_{\mathrm{C}_{\mathrm{MUL}}}=3 \times N
$$

The traditional method calculates the time-varying angular constraint by directly calculating the look angle in Equation (1). It should be noted that the resource occupation of the proposed scheme and the traditional method cannot be accurately compared because the implementation of arccosine is not unique. However, it is certain that, when the expansion order is the same, Equations (11), (13) and (14) contain additional division and sine modules compared to the proposed scheme. Therefore, the proposed scheme is simpler and more economical than that in Equation (11), when the expansion order is the same.

\section{Simulation and Analysis}

In this section, a spaceborne four-sub-swath STWE-SAR system is demonstrated to evaluate the performance of the proposed method. The parameters of the simulated DBF-SAR system are given in Table 1.

Table 1. Simulation system parameters.

\begin{tabular}{cc}
\hline Parameter & Value \\
\hline Carrier Frequency & $9.6 \mathrm{GHz}$ \\
Sample Rate & $1360 \mathrm{MHz}$ \\
Transmit Signal Pulse width & $10 \mu \mathrm{s}$ \\
Transmit Window Duration & $50 \mu \mathrm{s}$ \\
Signal Bandwidth & $600 \mathrm{MHz}$ \\
Orbit Height & $750 \mathrm{Km}$ \\
Reference Earth Radius & $6,371,393 \mathrm{~m}$ \\
Antenna Height (Elevation) & $2 \mathrm{~m}$ \\
Antenna Length (Azimuth) & $12 \mathrm{~m}$ \\
Number of Elevation Channels & 24 \\
Width of Sub-Swaths (Slant Range) & $80 \mathrm{Km}$ \\
Number of Sub-Swaths & 4 \\
\hline Ideal Resolution (Slant Range) & $0.25 \mathrm{~m}$ \\
Ideal Resolution (Azimuth) & $6 \mathrm{~m}$ \\
Total Witdh of Swath (Slant Range) & $320 \mathrm{Km}$ \\
\hline
\end{tabular}

Four sub-swaths intervals (marked in red) are illustrated in Figure 14. The timing diagram in Figure 14 shows the restrictions on the receive window due to transmit instances (marked in dark blue) and nadir echo (marked in light blue). 
The PRF and incident angle values of four sub-swaths are shown in Table 2. The total width of the four sub-swaths is $320 \mathrm{~km}$. The Doppler bandwidth is about $1250 \mathrm{~Hz}$, and the resolution in azimuth is about $6 \mathrm{~m}$. The resolution in the range is about $1 \mathrm{~m}$. The length of the receiving window is about $528 \mu$ s, corresponding to about 718,000 IF sampling points.

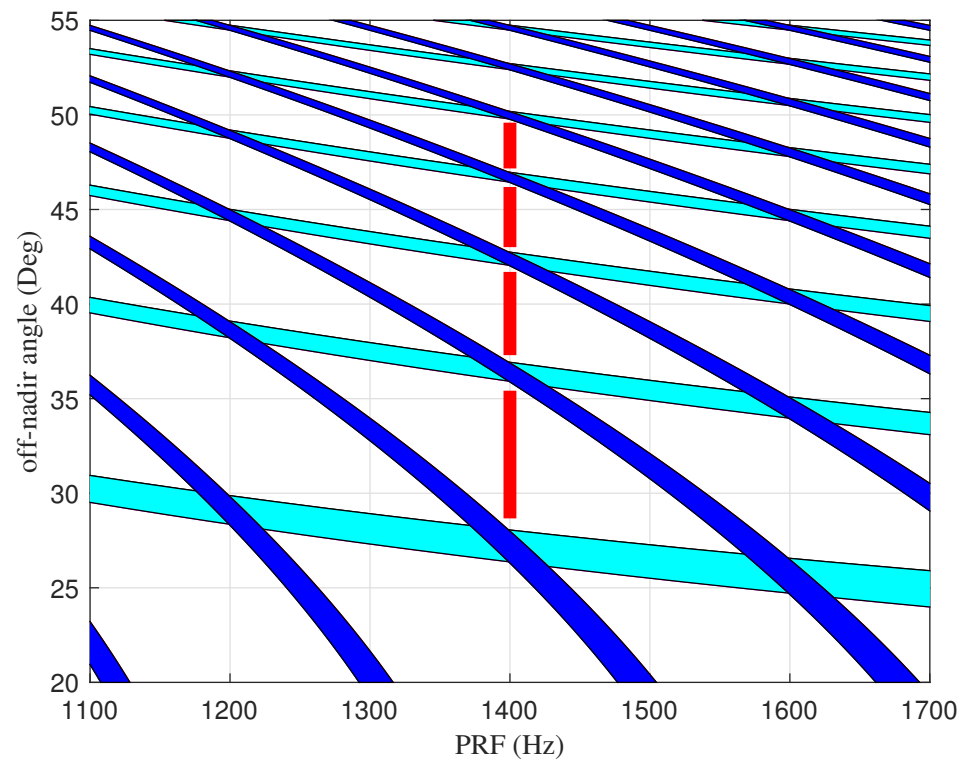

Figure 14. Timing diagram of the simulated STWE-SAR system.

Table 2. Information about four sub-swaths.

\begin{tabular}{ccc}
\hline Swath & PRF & Look Angle \\
\hline 1 & 1400 & {$\left[28.67^{\circ}, 35.42^{\circ}\right]$} \\
2 & 1400 & {$\left[37.30^{\circ}, 41.70^{\circ}\right]$} \\
3 & 1400 & {$\left[43.01^{\circ}, 46.19^{\circ}\right]$} \\
4 & 1400 & {$\left[47.17^{\circ}, 49.59^{\circ}\right]$} \\
\hline
\end{tabular}

The simulation flow is shown in Figure 15a. The echoes from four sub-swaths overlap each other in the time domain. The echoes are divided into four sub-echoes by the DBFbased echo separation technique. Imaging each subecho with common algorithms (e.g., RDA, CSA, wKA, etc.) yields to four independent images. The letters ' $X$ ' and ' $\mathrm{H}^{\prime}$ ', consisting of 13 point targets, are the targets of the simulation. The geometric relationships among the point targets is shown in Figure $15 \mathrm{~b}$.

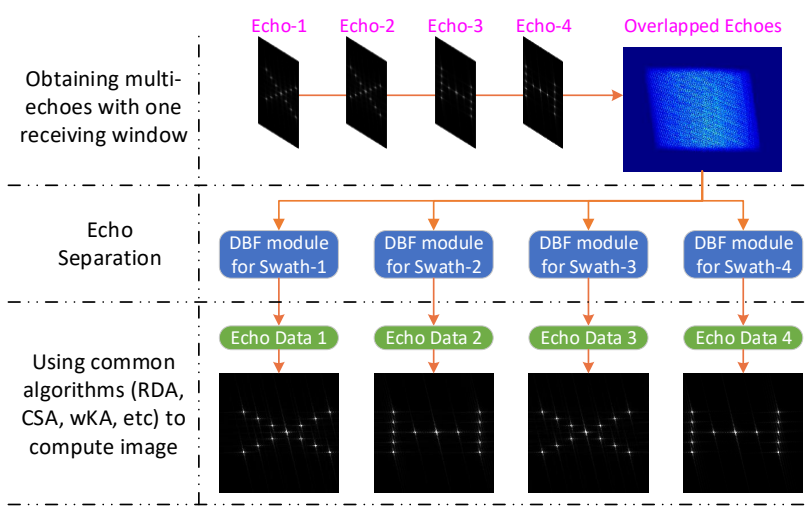

(a)

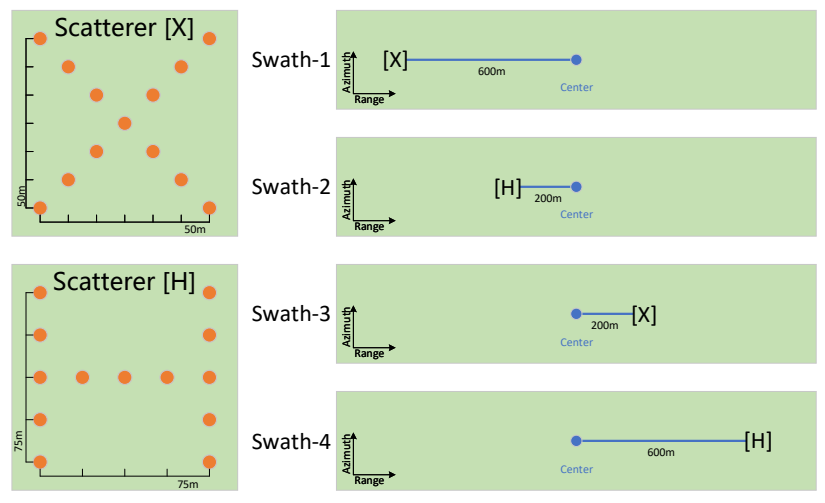

(b)

Figure 15. (a) The simulation flow. (b) The geometric relationship among the point targets. 


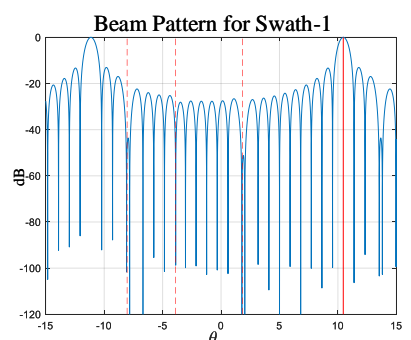

(a)

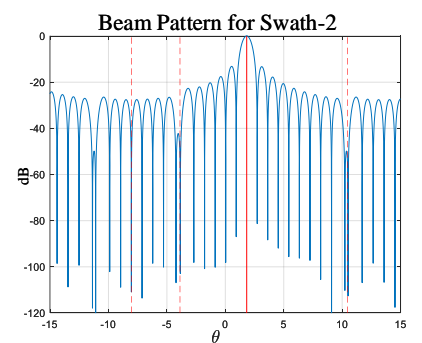

(b)
In STWE-SAR, onboard DBF modules generate time-varying beam patterns to achieve echo receiving and interference suppression simultaneously. The beam patterns generated by the conventional single-null constraint scheme for four sub-swaths are shown in Figure 16. The red solid line represents the main-lobe constraint, while the red dashed line represents the null constraint. It can be seen that although the gain level at the null point is below $-100 \mathrm{~dB}$, the gain level near the null point is relatively high. The gain level corresponding to the side-lobes do not provide a performance as at the null point, which will greatly reduce the interference suppression ability.

The beam patterns generated by the proposed multi-null constraint scheme for four subswaths are shown in Figure 17. Although the gain level corresponding to the notch is higher than the null point, it is much lower than the gain level corresponding to the side-lobes.

Figure 16. The beam patterns generated by the conventional single-null constraint scheme.

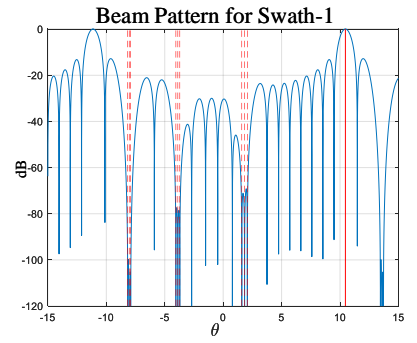

(a)

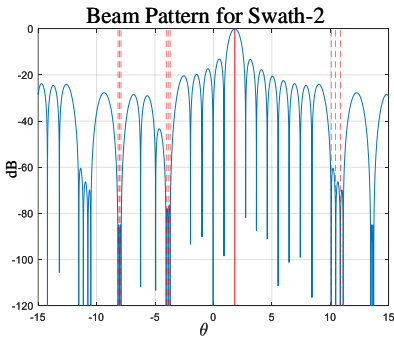

(b)

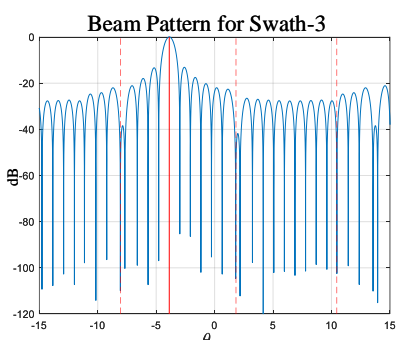

(c)

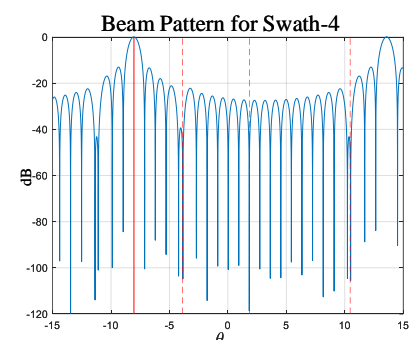

(d)

Figure 17. The beam patterns generated by the proposed multi-null constraint scheme.

The 2D simulation results are shown in Figure 18. In order to easily distinguish different point targets, a three-degree squint angle is introduced in the simulation. The four sub figures (a), (b), (c), and (d) on the left side in Figure 18 show the simulation results of the conventional single-null constrained scheme. Conventional schemes cannot completely suppress the interference from other sub-swaths. Although lower than the interested signal by $20 \sim 30 \mathrm{~dB}$, the interference energy is still evident after the range pulse compression. Since the range migration information and Doppler information of the interfering signal are different from the interested echo signal, the interfering signal cannot be compressed in azimuth. The interference energy produces bright curved lines along the azimuthal direction on SAR images.

The four figures (e), (f), (g), and (h) on the right side in Figure 18 show the simulation results for the proposed multi-null constraint scheme. The compressed interference energy greater than $-100 \mathrm{~dB}$ is not visible in any of the four images. The $2 \mathrm{D}$ simulation results clearly demonstrate the advantages of the proposed method compared to the conventional scheme. 


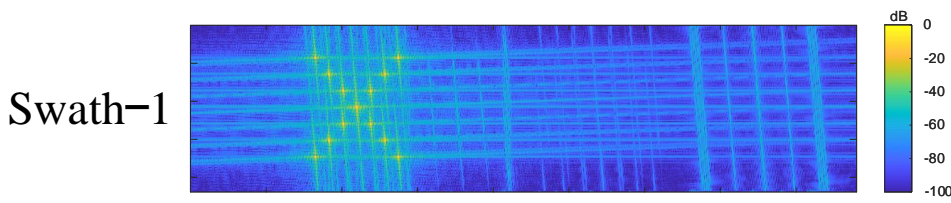

(a)

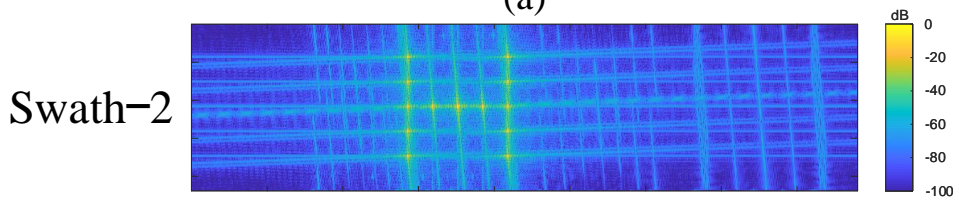

(b)

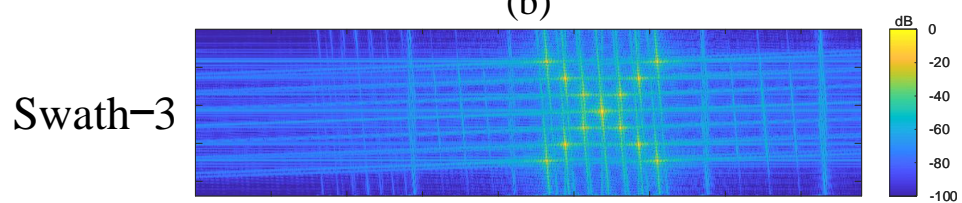

(c)

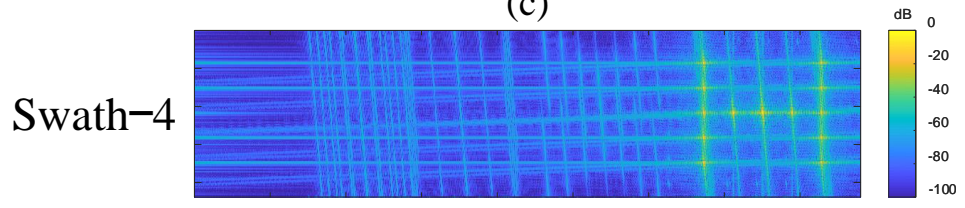

(d)

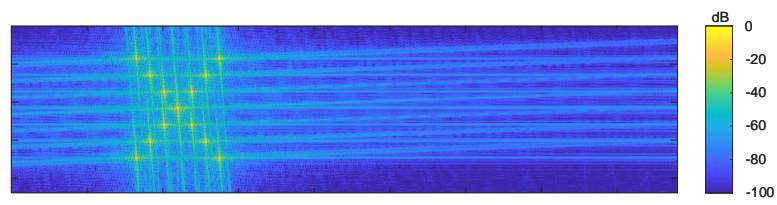

(e)

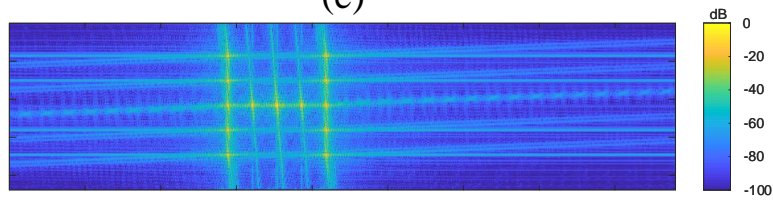

(f)

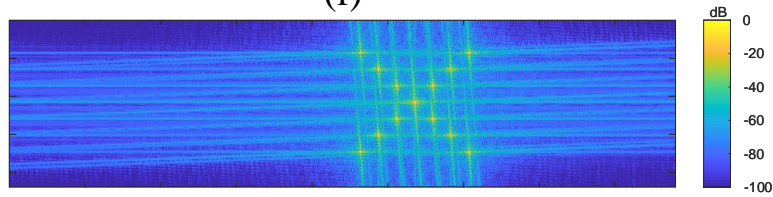

(g)

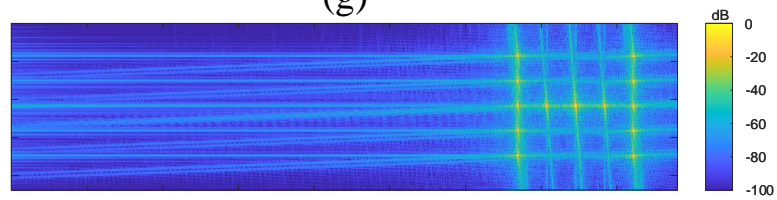

(h)

Figure 18. The 2D simulation results. (a-d) The 2D simulation results of the conventional single-null constrained scheme. (e-h) The 2D simulation results of the proposed multi-null constraint scheme.

To quantitatively analyze the effects of different orders of the null constraint on the interference suppression ability, the simulation results of NEL of sub-swath 1 corresponding to different orders are given in Figure 19. The first order null constraint corresponds to the conventional scheme. Simulation results of the third-order to the seventh-order multi-null constraints are evaluated. Since the beam pattern rotates with null constraints across multiple side-lobes, there are significant undulations in the NEL simulation results. Simulation results show that the NEL decreases significantly as the number of null orders increases. When the order is greater than 3 , each additional order of null constraint can reduce the NEL by about $30 \mathrm{~dB}$.

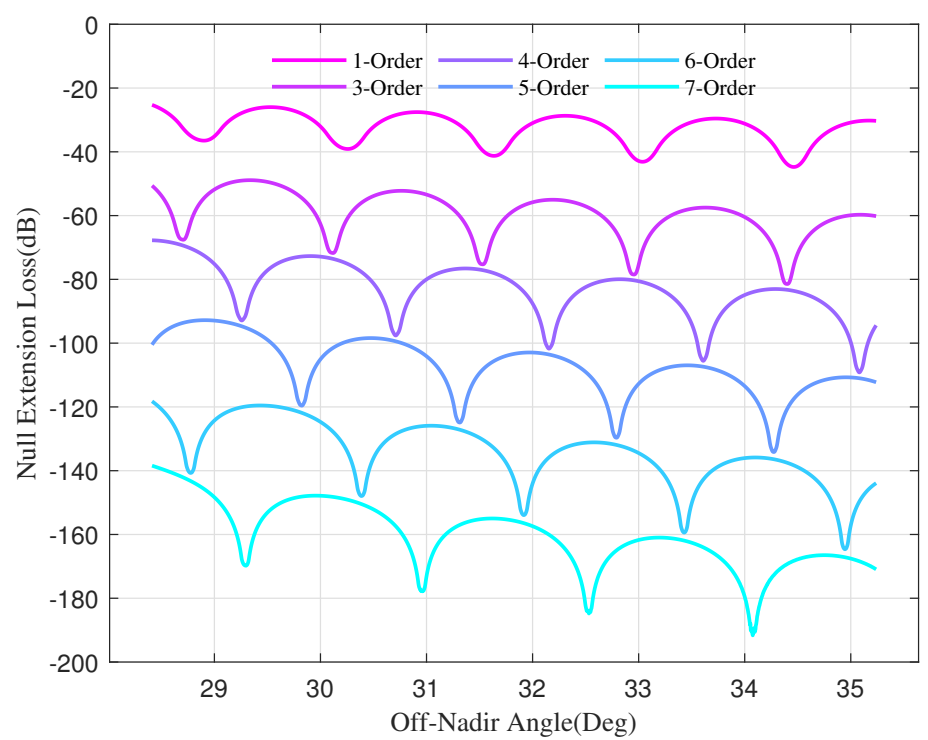

Figure 19. NEL effects corresponding to different null constraint orders for sub-swath 1. 
Table 3 gives the average NEL at different orders for different sub-swaths. The singlenull constraint corresponds to an average NEL comparable to the gain level of the side lobe, while the benefit of the multi-null constraint is obvious.

Table 3. The average NEL for four sub-swaths.

\begin{tabular}{ccccc}
\hline Order & Swath-1 & Swath-2 & Swath-3 & Swath-4 \\
\hline 1 & -32.8755 & -39.6801 & -43.2751 & -42.2908 \\
3 & -59.8992 & -74.5834 & -84.3336 & -88.5442 \\
4 & -83.4885 & -103.428 & -113.926 & -120.941 \\
5 & -107.704 & -130.451 & -145.837 & -153.970 \\
6 & -134.845 & -161.980 & -178.161 & -188.434 \\
7 & -161.084 & -188.833 & -182.322 & -184.303 \\
\hline
\end{tabular}

\section{Experimental Results}

The experiments were conducted using an X-band DBF-SAR system with 16 channels in the elevation to verify the effectiveness of the proposed scheme and real-time processing architecture. The raw data were collected by an experimental airborne SAR system, which was made by the Aerospace Information Research Institute, Chinese Academy of Sciences (AIRCAS). The system parameters of this SAR are given in Table 4 .

Table 4. Experimental System Parameters.

\begin{tabular}{cc}
\hline Parameter & Value \\
\hline Carrier frequency & $9.6 \mathrm{GHz}$ \\
Velocity & $70 \mathrm{~m} / \mathrm{s}$ \\
Height & $4200 \mathrm{~m}$ \\
Incident angle & $65^{\circ}$ \\
Signal bandwidth & $500 \mathrm{MHz}$ \\
Sampling frequency & $1200 \mathrm{MHz}$ \\
Signal pulse duration & $10 \mu \mathrm{s}$ \\
Number of elevation channels & 16 \\
\hline
\end{tabular}

Flight experiments were conducted in Guangdong Province, China. The data were collected in December 2019. The scenes were mainly composed of ponds, hills, farmland, and some man-made buildings. Alternating emissions were used to simulate the two sub-bands of the satellite in STWE imaging mode. Since the aircraft was much closer to the ground than the satellite, the echoes of the two scenes were not mixed during recording, but were stored on the disk in an odd-even frame format. The sampled data of the odd and even frames correspond to the echoes of both near-end and far-end sub-swaths, respectively. The echoes of the two scenes were mixed in the time domain before imaging, and then the overlapped echoes were separated into two sub-echoes by the DBF technique. The advantages of the proposed scheme over the conventional scheme can be compared by observing the imaging performance of the two sub-echoes separately.

The single-channel imaging results of the two scenes are shown in Figure 20, where (a) and (b) correspond to the two scenes in the near and far distance, respectively. Regions $A$, $B, C$, and D outline the man-made structures in figures (a) and (b).

Since the echoes from man-made structures are much stronger than natural features, strong point targets produce bright curves along the azimuth direction on the SAR image. Figure 21 illustrates the images of the conventional single-null constraint scheme, where significant interference energy can be seen in region A, B, C, and D. Figure 22 illustrates the images of the proposed multi-null constraint scheme. As shown in Figure 22, the proposed scheme effectively suppresses the energy of the interfering signal and the imaging performance is close to Figure 20. Due to non-ideal airborne systems and errors between channels, there is also residual interference energy that cannot be effectively suppressed. Relative to the ideal result in Figure 20, some weak interference energy can still be found in 
Figure 22. The experimental results visually demonstrate the advantages of the proposed method over the conventional scheme.

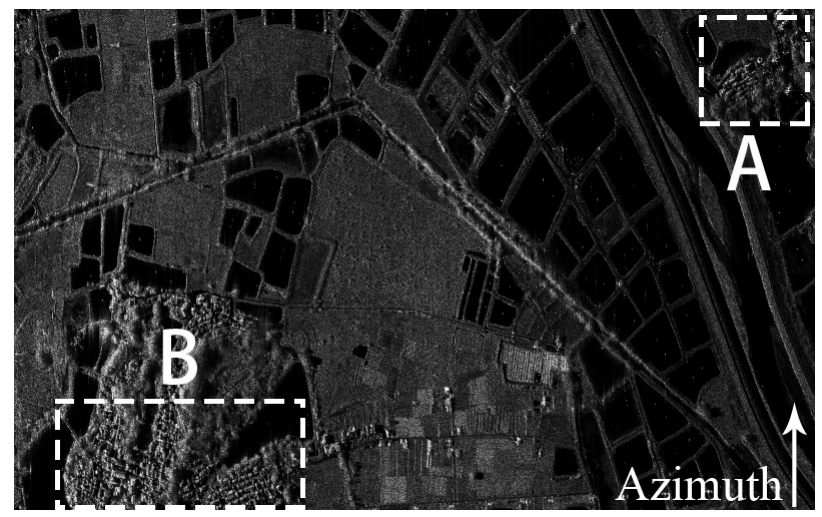

(a)

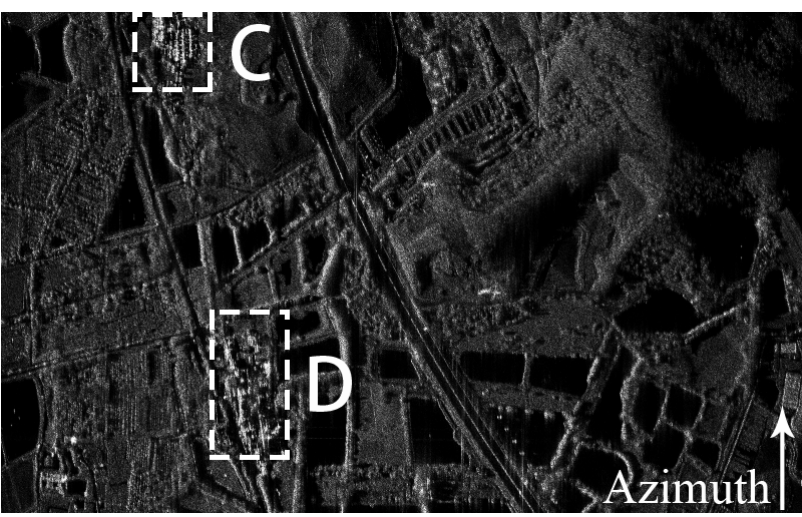

(b)

Figure 20. The single-channel images. (a) The near-end sub-swath. (b) The far-end sub-swath.

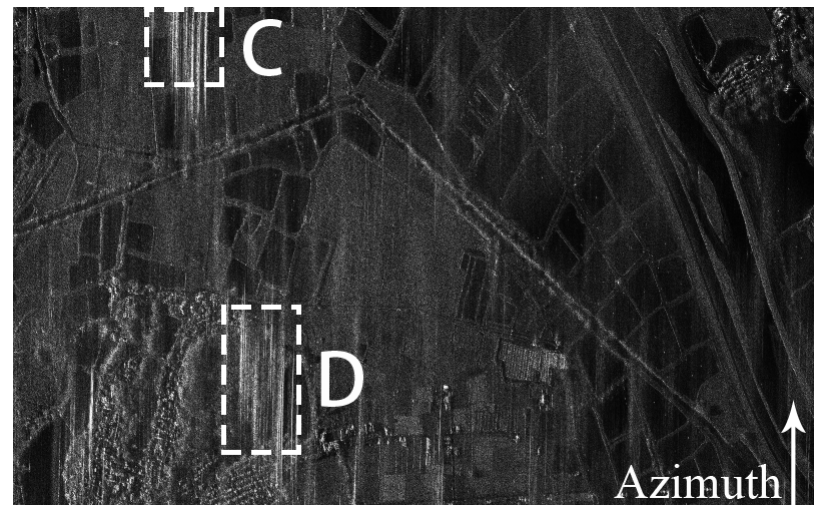

(a)

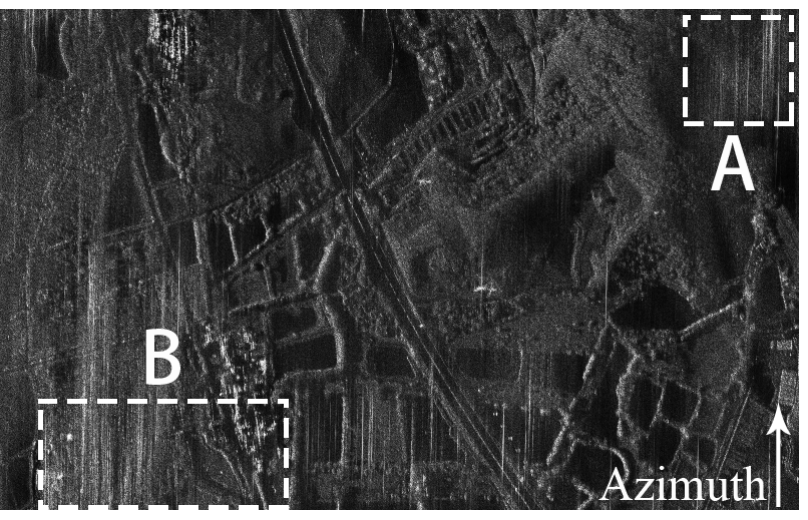

(b)

Figure 21. The images of the conventional scheme. (a) The near-end sub-swath. (b) The far-end sub-swath.

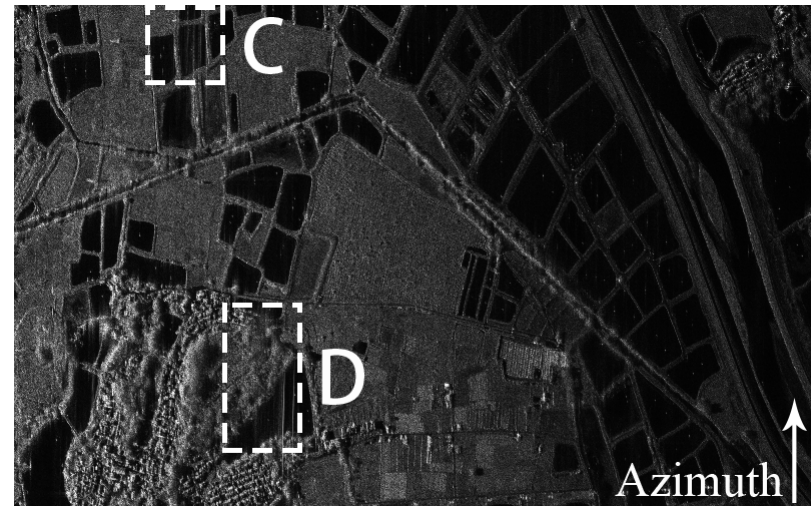

(a)

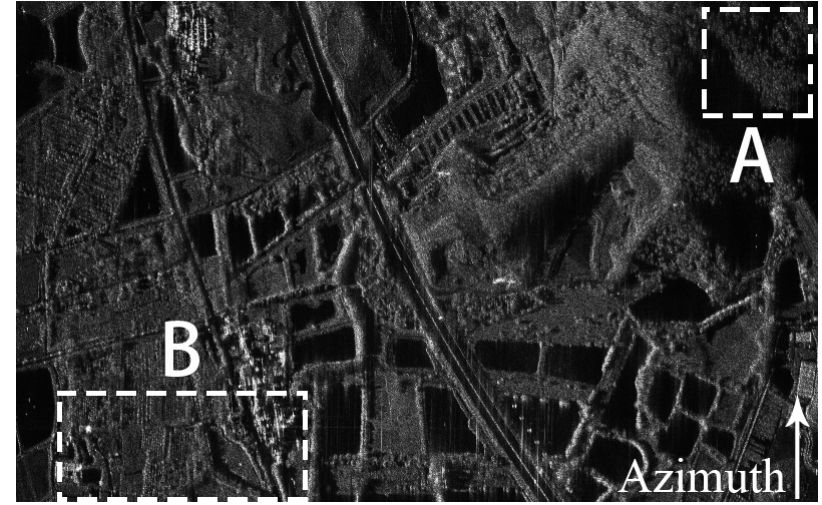

(b)

Figure 22. The images of the proposed scheme. (a) The near-end sub-swath. (b) The far-end sub-swath.

\section{Discussion}

The proposed scheme is one kind of hard real-time system. To be more accurate, the proposed scheme is not a computation method, but a calculation method. In other words, the weights are not computed by some programming language, but are calculated by a set of analytical expressions realized by timing logic circuits. The processing time 
of the system is synchronized with the sampling time of the echo. To ensure hard realtime requirements, pipelining techniques should be employed by FPGAs to enhance the processing time performance of operations.

In the STWE mode, the limitation of receiving multiple sub-swath echoes in the ScanSAR mode can be overcome. However, the signal transmission period would lead to a blind region. The largest continuous swath width is limited by pulse repetition frequency (PRF), pulse duration, transmitting window, etc. The so-called wide swath beyond that limited by PRF is noncontinuous and has blind regions, as the radar cannot receive echoes while transmitting [24]. If the transmitting and receiving antennas are separated, the blind region can be avoided.

In the traditional scheme, both the main lobe and the null modulate the envelope of the echo. The receiving gain loss due to the main lobe deviation from the target direction is no more than $-10 \mathrm{~dB}$. Due to the extremely narrow width of the null, most of the pulse energy is suppressed by side lobes. Simulation results show that the interference suppression capability of the null is greater than $-100 \mathrm{~dB}$. However, the interference suppression capability of the side lobe does not exceed $-40 \mathrm{~dB}$. Since the null can only cover a very small angle, most of the pulse energy is suppressed by side lobes. The interfering energy received by side lobes of the digital beam is still evident after range compression. Since the range migration information and Doppler information of the interfering signal are different from the interested echo signal, the interfering signal cannot be compressed in azimuth. The interference energy produces bright curved lines along the azimuthal direction on SAR images, as demonstrated in the 2D simulation in Section 4 and the experiments in Section 5. These bright lines have a greater impact on SAR images than range ambiguity energy.

Benefiting from the evolution of communication, smart antennas, MIMO, and other technologies, there are many inverse matrix implementations that can be adopted in the scheme proposed in this paper. For example, Givens rotations [25,26], Cholesky Decomposition [27], the Gauss-Jordan technique [28], LU decomposition [29], the Gram-Schmidt method [30], etc. Besides the above mentioned classical schemes, the Sherman-MorrisonWoodbury (SMW) formulas can be applied to compute the inverse matrix at every timestep in real-time [31]. The SMW formulas update the inverse of a matrix after a small perturbation, from the inverse of the original matrix. This method is normally employed in applications that apply consecutive perturbations to the matrix and require the inverse every time [32]. Inverting large matrices in real-time may lead to the non-convergence and a significant increase in complexity of the optimization problem. How to ensure the real-time of the DBF-SAR onboard processing will be further explored and solved in future research.

\section{Conclusions}

STWE is a potential technology used to achieve HRWS for the next generation of spaceborne DBF-SAR systems. Conventional schemes cannot effectively separate the echoes of multiple sub-swaths. Four significant problems (in the suppression of interfering energy in a space borne SAR) were analyzed. To overcome the shortcomings in the traditional scheme, a novel STWE real-time processing architecture is proposed in this paper. The proposed model has no special requirements for the orthogonality of the signal in the time or frequency domain. Based on the joint space-time processing, the time-domain envelope and spatial information of the signal were used to provide clues to the echo separation. The proposed algorithm is applicable for calculating the time-varying angular constraints in a general DBF real-time processing architecture. It can be applied to calculate not only null constraints, but also other time-varying angular constraints. In addition, the mathematical derivations and the real-time processing framework were discussed in detail. The simulation results show that the proposed scheme can effectively improve the performance of echo separation. The experimental results verify the effectiveness of the proposed method. In the future, we will continue to focus on implementing the SAR theory in practice. 
Author Contributions: Conceptualization, J.Q. and W.W.; methodology, J.Q.; validation, J.Q. and S.H.; formal analysis, J.Q.; investigation, Z.Z.; resources, Z.Z.; data curation, Z.C.; writing original draft preparation, J.Q. and Z.C.; writing review and editing, Y.W., X.M. and H.F. All authors have read and agreed to the published version of the manuscript.

Funding: This work was funded by the National Natural Science Foundation of China under grant numbers 61971401 and 61901442.

Institutional Review Board Statement: Not applicable.

Informed Consent Statement: Not applicable.

Data Availability Statement: Data sharing not applicable.

Conflicts of Interest: The authors declare no conflict of interest.

\section{Appendix A. The Exchange Matrix}

The exchange or reflection matrix $\mathbf{J}$ is a square $N \times N$ matrix, whose elements on the cross diagonal are unity and all other elements are zero.

Thus,

$$
\mathbf{J}=\left[\begin{array}{cccc}
0 & \cdots & 0 & 1 \\
0 & \cdots & 1 & 0 \\
\vdots & \ddots & \vdots & \vdots \\
1 & \cdots & 0 & 0
\end{array}\right]
$$

Appendix B. The Derivation Process of Equation (40)

In (36)

$$
C=\left\{\begin{array}{cc}
{\left[\begin{array}{c}
C_{S} \\
\hdashline 1 \\
\hdashline C_{S}^{*}
\end{array}\right],} & N \text { is odd } \\
{\left[\begin{array}{c}
C_{S} \\
\mathbf{J}_{S}^{*}
\end{array}\right],} & N \text { is even }
\end{array}\right.
$$

where

$$
C_{S}=\exp \left(-j\left[\begin{array}{cccc}
A F_{1} g_{\mathrm{b}} & A F_{1} g_{1} & \cdots & A F_{1} g_{\mathrm{Q}} \\
A F_{2} g_{\mathrm{b}} & A F_{2} g_{1} & \cdots & A F_{2} g_{\mathrm{Q}} \\
\vdots & \vdots & \ddots & \vdots \\
A F_{k} g_{\mathrm{b}} & A F_{k} g_{1} & \cdots & A F_{k} g_{\mathrm{Q}}
\end{array}\right]\right)
$$

and

$$
\mathbf{J} C_{s}^{*}=\exp \left(j\left[\begin{array}{cccc}
A F_{k} g_{b} & A F_{k} g_{1} & \cdots & A F_{k} g_{\mathrm{Q}} \\
\vdots & \vdots & \ddots & \vdots \\
A F_{2} g_{b} & A F_{2} g_{1} & \cdots & A F_{2} g_{\mathrm{Q}} \\
A F_{1} g_{b} & A F_{1} g_{1} & \cdots & A F_{1} g_{\mathrm{Q}}
\end{array}\right]\right)
$$

The term $\widetilde{(\bullet)}$ denotes the rotation of the matrix clockwise by 90 degrees. We have

$$
\widetilde{\boldsymbol{C}_{S}}=\exp \left(-j\left[\begin{array}{cccc}
A F_{k} g_{\mathrm{b}} & A F_{k-1} g_{\mathrm{b}} & \cdots & A F_{1} g_{\mathrm{b}} \\
A F_{k} g_{1} & A F_{k-1} g_{1} & \cdots & A F_{1} g_{1} \\
\vdots & \vdots & \ddots & \vdots \\
A F_{k} g_{\mathrm{Q}} & A F_{k-1} g_{\mathrm{Q}} & \cdots & A F_{1} g_{\mathrm{Q}}
\end{array}\right]\right)
$$


and

$$
\widetilde{\mathbf{J C}_{s}^{*}}=\exp \left(j\left[\begin{array}{cccc}
A F_{1} g_{b} & A F_{2} g_{b} & \cdots & A F_{k} g_{b} \\
A F_{1} g_{1} & A F_{2} g_{1} & \cdots & A F_{k} g_{1} \\
\vdots & \vdots & \ddots & \vdots \\
A F_{1} g_{\mathrm{Q}} & A F_{2} g_{\mathrm{Q}} & \cdots & A F_{k} g_{\mathrm{Q}}
\end{array}\right]\right)
$$

Obviously, we have

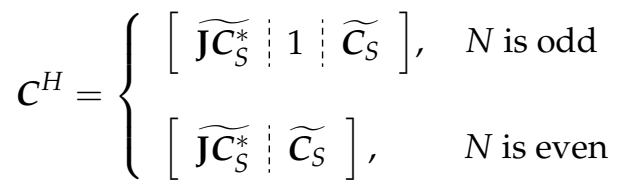

\section{References}

1. Huber, S.; de Almeida, F.Q.; Villano, M.; Younis, M.; Krieger, G.; Moreira, A. Tandem-L: A Technical Perspective on Future Spaceborne SAR Sensors for Earth Observation. IEEE Trans. Geosci. Remote Sens. 2018, 56, 4792-4807. [CrossRef]

2. Adamiuk, G.; Gabele, M.; Loinger, A.; Heer, C.; Ludwig, M. Technology demonstration for future DBF based spaceborne SAR missions. In Proceedings of the EUSAR 2018, 12th European Conference on Synthetic Aperture Radar, Aachen, Germany, 4-7 June 2018; pp. 1-6.

3. Bollian, T.; Osmanoglu, B.; Rincon, R.F.; Lee, S.K.; Fatoyinbo, T. MVDR Beamforming for RFI Suppression in EcoSAR data. In Proceedings of the EUSAR 2018, 12th European Conference on Synthetic Aperture Radar, Aachen, Germany, 4-7 June 2018; pp. 1-4.

4. Freeman, A.; Johnson, W.T.K.; Huneycutt, B.; Jordan, R.; Hensley, S.; Siqueira, P.; Curlander, J. The "Myth" of the minimum SAR antenna area constraint. IEEE Trans. Geosci. Remote Sens. 2000, 38, 320-324. [CrossRef]

5. Naftaly, U.; Levy-Nathansohn, R. Overview of the TECSAR Satellite Hardware and Mosaic Mode. IEEE Geosci. Remote Sens. Lett. 2008, 5, 423-426. [CrossRef]

6. Raney, R.; Luscombe, A.; Langham, E.; Ahmed, S. RADARSAT (SAR imaging). Proc. IEEE 1991, 79, 839-849. [CrossRef]

7. Gebert, N.; Krieger, G.; Moreira, A. Digital Beamforming on Receive: Techniques and Optimization Strategies for High-Resolution Wide-Swath SAR Imaging. IEEE Trans. Aerosp. Electron. Syst. 2009, 45, 564-592. [CrossRef]

8. Suess, M.; Grafmueller, B.; Zahn, R. A novel high resolution, wide swath SAR system. In Proceedings of the IGARSS 2001, Scanning the Present and Resolving the Future, IEEE 2001 International Geoscience and Remote Sensing Symposium (Cat. No.01CH37217), Sydney, Australia, 9-13 July 2001; Volume 3, pp. 1013-1015.

9. Zhou, Y.; Wang, W.; Chen, Z.; Wang, P.; Zhang, H.; Qiu, J.; Zhao, Q.; Deng, Y.; Zhang, Z.; Yu, W.; et al. Digital Beamforming Synthetic Aperture Radar (DBSAR): Experiments and Performance Analysis in Support of 16-Channel Airborne X-Band SAR Data. IEEE Trans. Geosci. Remote Sens. 2021, 59, 6784-6798. [CrossRef]

10. Feng, F.; Li, S.; Yu, W.; Huang, P.; Xu, W. Echo Separation in Multidimensional Waveform Encoding SAR Remote Sensing Using an Advanced Null-Steering Beamformer. IEEE Trans. Geosci. Remote Sens. 2012, 50, 4157-4172. [CrossRef]

11. Zhao, Q.; Zhang, Y.; Wang, W.; Deng, Y.; Yu, W.; Zhou, Y.; Wang, R. Echo Separation for Space-Time Waveform-Encoding SAR With Digital Scalloped Beamforming and Adaptive Multiple Null-Steering. IEEE Geosci. Remote Sens. Lett. 2021, 18, 92-96. [CrossRef]

12. Krieger, G.; Gebert, N.; Moreira, A. Multidimensional Waveform Encoding: A New Digital Beamforming Technique for Synthetic Aperture Radar Remote Sensing. IEEE Trans. Geosci. Remote Sens. 2008, 46, 31-46. [CrossRef]

13. Fischer, C.; Heer, C.; Werninghaus, R. X-Band HRWS demonstrator: Digital Beamforming test results. In Proceedings of the EUSAR 2012, 9th European Conference on Synthetic Aperture Radar, Nuremberg, Germany, 23-26 April 2012; pp. 1-4.

14. Feng, F.; Dang, H.; Tan, X.; Li, G.; Li, C. An improved scheme of Digital Beam-Forming in elevation for spaceborne SAR. In Proceedings of the IET International Radar Conference 2013, Guilin, China, 14-16 April 2013; pp. 1-6.

15. Younis, M.; Rommel, T.; Bordoni, F.; Krieger, G.; Moreira, A. On the Pulse Extension Loss in Digital Beamforming SAR. IEEE Geosci. Remote Sens. Lett. 2015, 12, 1436-1440. [CrossRef]

16. Wang, W.; Wang, R.; Deng, Y.; Balz, T.; Hong, F.; Xu, W. An Improved Processing Scheme of Digital Beam-Forming in Elevation for Reducing Resource Occupation. IEEE Geosci. Remote Sens. Lett. 2016, 13, 309-313. [CrossRef]

17. Qiu, J.; Zhang, Z.; Wang, R.; Wang, P.; Zhang, H.; Du, J.; Wang, W.; Chen, Z.; Zhou, Y.; Jia, H.; et al. A Novel Weight Generator in Real-Time Processing Architecture of DBF-SAR. IEEE Trans. Geosci. Remote Sens. 2021. [CrossRef]

18. Zhao, Q.; Zhang, Y.; Wang, W.; Liu, K.; Deng, Y.; Zhang, H.; Wang, Y.; Zhou, Y.; Wang, R. On the Frequency Dispersion in DBF SAR and Digital Scalloped Beamforming. IEEE Trans. Geosci. Remote Sens. 2020, 58, 3619-3632. [CrossRef]

19. Van Trees, H.L. Optimum Array Processing: Part IV of Detection, Estimation, and Modulation Theory; John Wiley \& Sons, Inc.: New York, NY, USA, 2002. 
20. Mizzoni, R.; Capece, P.; Contu, S.; Meschini, A.; Ivagnes, M.; Rosati, G. Antennas for observation, exploration and navigation in ThalesAleniaSpace-Italia: Past and present challenges. In Proceedings of the 2017 11th European Conference on Antennas and Propagation (EUCAP), Paris, France, 19-24 March 2017; pp. 1516-1520. [CrossRef]

21. Volder, J.E. The CORDIC Trigonometric Computing Technique. IRE Trans. Electron. Comput. 1959, EC-8, 330-334. [CrossRef]

22. Irturk, A.; Benson, B.; Mirzaei, S.; Kastner, R. An FPGA Design Space Exploration Tool for Matrix Inversion Architectures. In Proceedings of the 2008 Symposium on Application Specific Processors(SASP), Anaheim, CA, USA, 8-9 June 2008; pp. 42-47. [CrossRef]

23. de Almeida, F.Q.; Younis, M.; Krieger, G.; Hensley, S.; Moreira, A. Investigation into the Weight Update Rate for Scan-On-Receive Beamforming. In Proceedings of the 2020 IEEE Radar Conference (RadarConf20), Florence, Italy, 21-25 September 2020; pp. 1-6. [CrossRef]

24. Yang, T.; Lv, X.; Wang, Y.; Qian, J. Study on a Novel Multiple Elevation Beam Technique for HRWS SAR System. IEEE J. Sel. Top. Appl. Earth Obs. Remote Sens. 2015, 8, 5030-5039. [CrossRef]

25. Echman, F.; Owall, V. A scalable pipelined complex valued matrix inversion architecture. In Proceedings of the 2005 IEEE International Symposium on Circuits and Systems (ISCAS), Kobe, Japan, 23-26 May 2005; Volume 5, pp. 4489-4492. [CrossRef]

26. Ma, L.; Dickson, K.; McAllister, J.; McCanny, J. Modified givens rotations and their application to matrix inversion. In Proceedings of the 2008 IEEE International Conference on Acoustics, Speech and Signal Processing, Las Vegas, Nevada, USA, 30 March-4 April 2008; pp. 1437-1440. [CrossRef]

27. Singh, A.K. Fast inversion of positive definite Hermitian matrices using real inverse operations. In Proceedings of the 2015 Annual IEEE India Conference (INDICON), New Delhi, India, 17-20 December 2015; pp. 1-3. [CrossRef]

28. V, C.; Kuloor, R. Novel Architecture for Highly Hardware Efficient Implementation of Real Time Matrix Inversion Using Gauss Jordan Technique. In Proceedings of the 2010 IEEE Computer Society Annual Symposium on VLSI, Lixouri, Greece, 5-7 July 2010; pp. 294-298. [CrossRef]

29. Khan, F.A.; Ashraf, R.A.; Abbasi, Q.H.; Nasir, A.A. Resource efficient parallel architectures for linear matrix algebra in real time adaptive control algorithms on reconfigurable logic. In Proceedings of the 2008 Second International Conference on Electrical Engineering, Lahore, Pakistan, 25-26 March 2008; pp. 1-9. [CrossRef]

30. Rosado, A.; Iakymchuk, T.; Bataller, M.; Wegrzyn, M. Hardware-efficient matrix inversion algorithm for complex adaptive systems. In Proceedings of the 2012 19th IEEE International Conference on Electronics, Circuits, and Systems (ICECS 2012), Seville, Spain, 9-12 December 2012; pp. 41-44. [CrossRef]

31. Hager, W.W. Updating the Inverse of a Matrix. SIAM Rev. 1989, 31, 221-239. [CrossRef]

32. Hadizadeh, A.; Hashemi, M.; Labbaf, M.; Parniani, M. A Matrix-Inversion Technique for FPGA-Based Real-Time EMT Simulation of Power Converters. IEEE Trans. Ind. Electron. 2019, 66, 1224-1234. [CrossRef] 